\title{
Outflows and Massive Stars in the protocluster IRAS 05358+3543
}

\author{
Adam G. Ginsburg and John Bally \\ Center for Astrophysics and Space Astronomy, Department of Astrophysical and Planetary \\ Sciences \\ University of Colorado \\ 389 UCB, Boulder, CO 80309-0389 \\ Adam.Ginsburg@colorado.edu \\ John.Bally@colorado.edu \\ Chi-Hung Yan \\ Institute of Astronomy and Astrophysics, Academia Sinica \\ P.O. Box 23-141, Taipei, Taiwan \\ Department of Earth Sciences, National Taiwan Normal University \\ \#88 Sec. 4, Ting-chow Rd., Taipei, Taiwan \\ chyan@asiaa.sinica.edu.tw \\ Jonathan P. Williams \\ Institute for Astronomy \\ University of Hawaii \\ 2680 Woodlawn Dr. Honolulu, HI 96822
}

\begin{abstract}
We present new near-IR $\mathrm{H}_{2}, \mathrm{CO} \mathrm{J}=2-1$, and $\mathrm{CO} \mathrm{J}=3-2$ observations to study outflows in the massive star forming region IRAS 05358+3543. The Canada-FranceHawaii Telescope $\mathrm{H}_{2}$ images and James Clerk Maxwell Telescope CO data cubes of the IRAS 05358 region reveal several new outflows, most of which emerge from the dense cluster of sub-mm cores associated with the Sh 2-233IR NE cluster to the northeast of IRAS 05358. We used Apache Point Observatory (APO) JHK spectra to determine line of sight velocities of the outflowing material. Analysis of archival VLA cm continuum data and previously published VLBI observations reveal a massive star binary as a probable source of one or two of the outflows. We have identified probable sources for
\end{abstract}


6 outflows and candidate counterflows for 7 out of a total of 11 seen to be originating from the IRAS 05358 clusters. We classify the clumps within Sh 2-233IR NE as an early protocluster and Sh 2-233IR SW as a young cluster, and conclude that the outflow energy injection rate approximately matches the turbulent decay rate in Sh 2-233IR NE.

Subject headings: ISM: jets and outflows — ISM: Herbig-Haro objects — ISM: kinematics and dynamics — ISM: individual: S233IR — stars: formation

\section{Introduction}

Collimated, bipolar outflows accompany the birth of young stars from the earliest stages of star formation to the end of their accretion phase (e.g. Reipurth \& Bally 2001). While the birth of isolated low-mass stars is becoming well understood, the formation of massive stars $\left(>10 M_{\odot}\right)$ and clusters remains a topic of intense study. Observations show that moderate to high-mass stars tend to form in dense clusters (Lada \& Lada 2003). In a clustered environment, the dynamics of the gas and stars can profoundly impact both accretion and mass-loss processes. Feedback from these massive clusters may play a significant role in momentum injection and turbulence driving in the interstellar medium.

Outflows from massive stars are less studied than those from low mass stars largely because massive stars accrete most of their mass while deeply embedded. Therefore, unlike low mass young stars that are accessible in the optical, massive stellar outflows can only be seen at infrared and longer wavelengths. Direct evidence for jets from massive young stellar objects (YSOs) from $\mathrm{H}_{2}$ or optical emission is generally lacking (e.g. Alvarez \& Hoare 2005; Kumar et al. 2002; Wang et al. 2003), although there is evidence that massive stars are the sources of collimated molecular outflows from millimeter observations (e.g. Beuther et al. 2002b). Outflows from massive stars may allow accretion to continue after their radiation pressure would otherwise halt accretion in a spherically symmetric system (Krumholz et al. 2009). They therefore represent a crucial component in understanding how stars above $\sim 10 M_{\odot}$ can form.

IRAS 05358 is a double cluster of embedded infrared sources located at a distance of $1.8 \mathrm{kpc}$ in the Auriga molecular cloud complex (Heyer et al. 1996) associated with the HII regions Sh-2 231 through 235 at Galactic coordinates around $l, b=173.48,+2.45$ in the Perseus arm. Sh 2-233IR NE is the collection of highly obscured and mm-bright sources slightly northeast of Sh 2-233IR SW, which is the location of the IRAS $05358+3543$ point source and the optical emission nebula (see Figure 1). The IRAS source is probably a blend of the three brightest infrared objects in the MSX A-band and MIPS $24 \mu \mathrm{m}$ images, which are located at Sh 2-233IR NE, IR 41, and IR 6. For the purpose of this paper,the whole complex including both sources is referred toas IRAS 05358, and otherwise refer to individual objects specifically.

Early observations revealed the presence of $\mathrm{OH}$ (Wouterloot et al. 1993), $\mathrm{H}_{2} \mathrm{O}$ (Scalise et al. 
1989; Henning et al. 1992), and methanol (Menten 1991) masers about an arcminute northeast of the IRAS source, indicating that massive stars are likely present at that location. Near infrared observations revealed the presence of two embedded clusters (Porras et al. 2000; Jiang et al. 2001) labeled Sh 2-233IR SW for the southwestern cluster associated with the IRAS source, and Sh 2233IR NE for the northeastern cluster located near the $\mathrm{OH}, \mathrm{H}_{2} \mathrm{O}$, and $\mathrm{CH}_{3} \mathrm{OH}$ masers. Stars identified in Porras et al. (2000) are referred to by the designation "IR (number)" corresponding to the catalog number in that paper. Porras et al. (2000) also included scanning Fabry-Perot velocity measurements of the inner $\sim 1^{\prime}$. CO observations revealed broad line wings indicative of a molecular outflow (Casoli et al. 1986; Shepherd \& Churchwell 1996). Kumar et al. (2002) and Khanzadyan et al. (2004) presented narrow band images of $2.12 \mu \mathrm{m} \mathrm{H}_{2}$ emission that reveled the presence of multiple outflows. Interferometric imaging of $\mathrm{CO}$ and $\mathrm{SiO}$ confirmed the presence of at least three flows emerging from the northeast cluster centered on the masers (Beuther et al. 2002a) having a total mass of about $20 M_{\odot}$. Beuther et al. (2002a) also presented MAMBO 1.2 mm maps and a mass estimate of $610 M_{\odot}$ for the whole region. Williams et al. (2004) presented SCUBA maps and mass estimates of the clusters of $195 / 126 M_{\odot}$ for Sh 2-233IR NE and 24/12 $M_{\odot}$ for Sh 2-233IR SW $(850 \mu \mathrm{m} / 450 \mu \mathrm{m})$. Zinchenko et al. (1997) measured the dense gas properties using the $\mathrm{NH}_{3}(1,1)$ and $(2,2)$ lines. They measure a mean density $n \approx 10^{3.60} \mathrm{~cm}^{-3}$, temperature $26.5 \mathrm{~K}$, and a mass of $600 M_{\odot}$. The total luminosity of the two clusters is about $6300 L_{\odot}$, indicating that the region is giving birth to massive stars (Porras et al. 2000).

Millimeter wavelength interferometry with arcsecond angular resolution has revealed a compact cluster of deeply embedded sources centered on the $\mathrm{H}_{2} \mathrm{O}$ and methanol maser position (Beuther et al. 2002a, 2007; Leurini et al. 2007). Beuther et al. (2002a) identified $3 \mathrm{~mm}$ continuum cores, labeled mm1-mm3 (shown in Figure 2). Beuther et al. (2007) resolved these cores into smaller objects. Source mm1a is associated with a cm continuum point source and will be discussed in detail below.

IRAS 05358 has previously been observed at low spatial resolution in the $\mathrm{J}=2-1$ and $\mathrm{J}=3-2$ transitions with the Kosma 3m telescope (Mao \& Zeng 2004). While the general presence of outflows was recognized and a total mass estimated, the specific outflows were not resolved. Beuther et al.

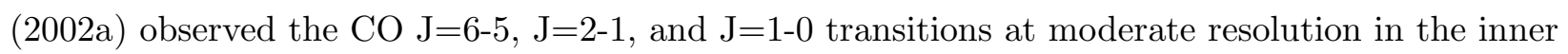
few arcminutes. Thomas \& Fuller (2008) observed $\mathrm{C}^{17} \mathrm{O}$ in the $\mathrm{J}=2-1$ and $\mathrm{J}=3-2$ transitions with a single pointing using the JCMT.

\section{Observations}

A collection of data acquired by the authors and from publicly available archives is presented. An overview of the data is presented in figure 1. The goal was to develop a complete picture of the outflows in IRAS 05358 and their probable sources. CO data were acquired to estimate the total outflowing mass and to identify outflowing molecular material unassociated with $\mathrm{H}_{2}$ shocks. Archival Spitzer IRAC and MIPS $24 \mu \mathrm{m}$ data were used to identify probable YSOs as candidate outflow sources. Near-infrared spectra were acquired primarily to determine $\mathrm{H}_{2}$ kinematics and 
develop a 3D picture of the region. Optical spectra were acquired to attempt to identify stellar types in the unobscured Sh 2-233IR SW region. Finally, archival VLA data were used to acquire better constraints on the position and physical properties of the known ultracompact HII (UCHII) region, and to detect or set limits on other UCHIIs.

\subsection{Sub-millimeter Observations}

The $345 \mathrm{GHz} \mathrm{J}=3-2$ rotational transition of $\mathrm{CO}$ was observed with the James Clerk Maxwell Telescope (JCMT) on 4 January, 2008 with the 16 element (14 functional) HARP-B heterodyne focal plane array. Two $12^{\prime} \times 10^{\prime}$ raster scans in R.A. and Dec. were taken with orthogonal orientations to assure complete coverage in the region of interest; this resulted in a useable field $11.7^{\prime} \times 11.3^{\prime}$ with higher noise along the edges. The beam size at $345 \mathrm{GHz}$ is about $15^{\prime \prime}$.

Observations were conducted during grade 3 conditions with the $225 \mathrm{GHz}$ zenith optical depth of the atmosphere $\tau \sim 0.1$. A channel width of $488 \mathrm{kHz}$ corresponding to $0.423 \mathrm{~km} \mathrm{~s}^{-1}$ was used. The maps required a total of 1 hour to acquire and resulted in an effective integration time of 4.6 seconds per pixel (there are 12,000 $6 \times 6^{\prime \prime}$ pixels in the final grid), resulting in a noise per pixel of $0.36 \mathrm{~K} \mathrm{~km} \mathrm{~s}^{-1}$.

The optical depth and telescope efficiency corrections were applied by the JCMT pipeline to convert the recorded antenna temperatures to the corrected antenna 1 . An additional main-beam correction has been applied,

$$
T_{m b}=\frac{T_{A} *}{\eta_{m b}}
$$

where $\eta_{m b}$ was measured by observing Mars to be $\approx 0.60$ at $345 \mathrm{GHz}$. Emission in the sidelobes is expected to be small at the outflow velocities.

On September 25 and November 15, 2008 the CO, ${ }^{13} \mathrm{CO}$, and $\mathrm{C}^{18} \mathrm{O} \mathrm{J}=2-1$ transitions were observed in the central $3^{\prime}$ of IRAS 05358 . The beamsize at $220 \mathrm{GHz}$ is about $23^{\prime \prime}$. The sideband configuration used also the SO $5_{6}-4_{5}$ and ${ }^{13} \mathrm{CS} 5-4$ transitions. Conditions during these observations were grade $5(\tau \sim$ $0.24-0.28)$ and therefore too poor to use the HARP instrument, but acceptable for the A3 detector.

Data reduction used the Starlink package following the standard routines recommended by the JCMT support scientists 2. The CO 3-2 data cube was extracted over a velocity range from -50 to $10 \mathrm{~km} \mathrm{~s}^{-1}$ LSR and spectral baselines were fit over the velocity range -50 to -40 and 0 to $10 \mathrm{~km} \mathrm{~s}^{-1}$ and subtracted. The data were re-gridded into $6^{\prime \prime}$ pixels and 2 pixel Gaussian smoothing was used

\footnotetext{
${ }^{1}$ See

http://docs.jach.hawaii.edu/JCMT/OVERVIEW/tel_overview/ for a discussion of JCMT parameters

2 http://www.jach.hawaii.edu/JCMT/spectral_line/data_reduction/acsisdr/
} 
to fill in the gaps left by the two bad detectors in the $4 \times 4$ array. The data cube was cropped to remove undersampled edges which have high noise and bad baselines. The beam efficiency was 0.68 at $230 \mathrm{GHz}$.

The A3 data cubes were extracted over the velocity range -60 to $20 \mathrm{~km} \mathrm{~s}^{-1}$ and baselines were calculated over -60 to -40 and 0 to $20 \mathrm{~km} \mathrm{~s}^{-1}$. The data was gridded into $10^{\prime \prime}$ pixels with 2 pixel gaussian smoothing to reduce sub-resolution noise variations.

\subsection{Spitzer}

Spitzer IRAC bands 1 to 4 and MIPS band 1 data were retrieved from the Spitzer Science Center archive. Qiu et al. (2008) acquired the data as part of a study of many high-mass star forming regions; they identified YSO candidates based on IRAC colors. The version 18 post-BCD data products were used to produce images and photometric catalog from Qiu et al. (2008), which was made from a more carefully-reduced data set, was used for SED analysis.

\subsection{Near-IR images}

Near-infrared data were acquired using the Wide-field Infrared Camera (WIRCam) on the Canada-France-Hawaii Telescope (CFHT) on Mauna Kea. The field of view is $20^{\prime} \times 20^{\prime}$ and pixel scale $0.3^{\prime \prime}$. Data were acquired on November 18, 19 and December 20, 2005. The seeing was 0.5-0.7" during the observations. A $0.032 \mu \mathrm{m}$ wide filter centered at $2.122 \mu \mathrm{m}$ was used to take images of the $\mathrm{H}_{2} \mathrm{~S}(1)$ 1-0 rovibrational transition. Each $\mathrm{H}_{2}$ exposure was 58 seconds, and dithered images were taken for a total exposure time of 1755 seconds. The data were reduced with the WIRCam pipeline.

\subsection{Near-IR spectra}

Near-infrared spectra were acquired using the TripleSpec instrument at Apache Point Observatory. TripleSpec simultaneously acquires J, H, and K band spectra over a $42^{\prime \prime}$ long slit. A slit width of $1.1^{\prime \prime}$ with an approximate spectral resolution $\lambda / \Delta \lambda=2700$ was used.

Observations were taken on the nights of December 2, 2008 and January 7, 2009. Data on December 2 were taken in an ABBA nod pattern, but because of the need to observe extended structure across the slit a stare strategy was selected on January 7.

The data were reduced using the TwODSPEC package in IRAF. HD31135, an A0 star, was used as a flux calibrator. Wavelength calibration was performed using night sky lines. Lines filling the

slit were subtracted to remove atmospheric emission lines. Telluric absorption correction was not 
performed, but telluric absorption is considered in the analysis.

The transformations from the observed geocentric reference frame to $v_{L S R}$ were computed to be $0.78 \mathrm{~km} \mathrm{~s}^{-1}$ on Dec 2 and $19.74 \mathrm{~km} \mathrm{~s}^{-1}$ on Jan 8 .

\subsection{Optical Spectra}

Optical spectra were acquired using the Double Imaging Spectrograph instrument at APO. The high-resolution red and blue gratings were centered at $6564 \AA$ and $5007 \AA$ with a coverage of about 1200 angstroms and resolution $\lambda / \Delta \lambda \approx 5000$. Sets of three 900 s exposures and three $200 \mathrm{~s}$ exposures were acquired on the targets and on the spectrophotometric calibrator G191-b2b with a 1.5" slit. Observations were taken on the night of January 17, 2009 under clear conditions.

Optical spectra were also reduced using the TWODSPEC package in IRAF. Wavelength calibration was done with HeNeAr lamps and night sky lines in the red band, and HeNeAr lamps in the blue band. Lines filling the slit were subtracted to remove atmospheric lines, though some astrophysical lines also filled the slit and these were measured before background subtraction. The $v_{L S R}$ correction for this date was $24.4 \mathrm{~km} \mathrm{~s}^{-1}$.

\subsection{Optical imaging}

CCD images images were obtained on the nights of 14 and 15 September 2009 NOAO Mosaic 1 Camera at the f/3.1 prime focus of the 4 meter Mayal telescope atthe Kitt Peak National Observatory (KPNO). The Mosaic 1 camera is a $8192 \times 8192$ pixel array (consisting of eight $2048 \times 4096$ pixel CCD chips) with a pixel scale of $0.26^{\prime \prime}$ pixel $^{-1}$ and a field of view $35.4^{\prime}$ on a side. Narrow-band filters centered on $6569 \AA$ and $6730 \AA$ both with a FWHM of $80 \AA$ were use to obtain $\mathrm{H} \alpha$ and [SII] images. An SDSS i' filter which is centered on $7732 \AA$ with a FWHM of $1548 \AA$ was used for continuum imaging. A set of five dithered 600 second exposures were obtained in $\mathrm{H} \alpha$ and [SII] using the standard MOSDITHER pattern to eliminate cosmic rays and the gaps between the individual chips in Mosaic. A dithered set of five 180 second exposures were obtained in the in the broad-band SDSS i-band filter to discriminate between $\mathrm{H} \alpha$, [SII], and continuum emission. Images were reduced in the standard manner by the NOAO Mosaic reduction pipeline (Valdes \& Swaters 2007).

\subsection{VLA data}

VLA archival data from projects AR482, AR513, AS831, and AM697 were re-reduced to perform a deeper search for UCHII regions and aquire more data points on the known UCHII's SED. Data from AR482 were previously published in Beuther et al. (2007), the other data are unpublished. The data were reduced using the VLA pipeline in AIPS (VLARUN). The observations 


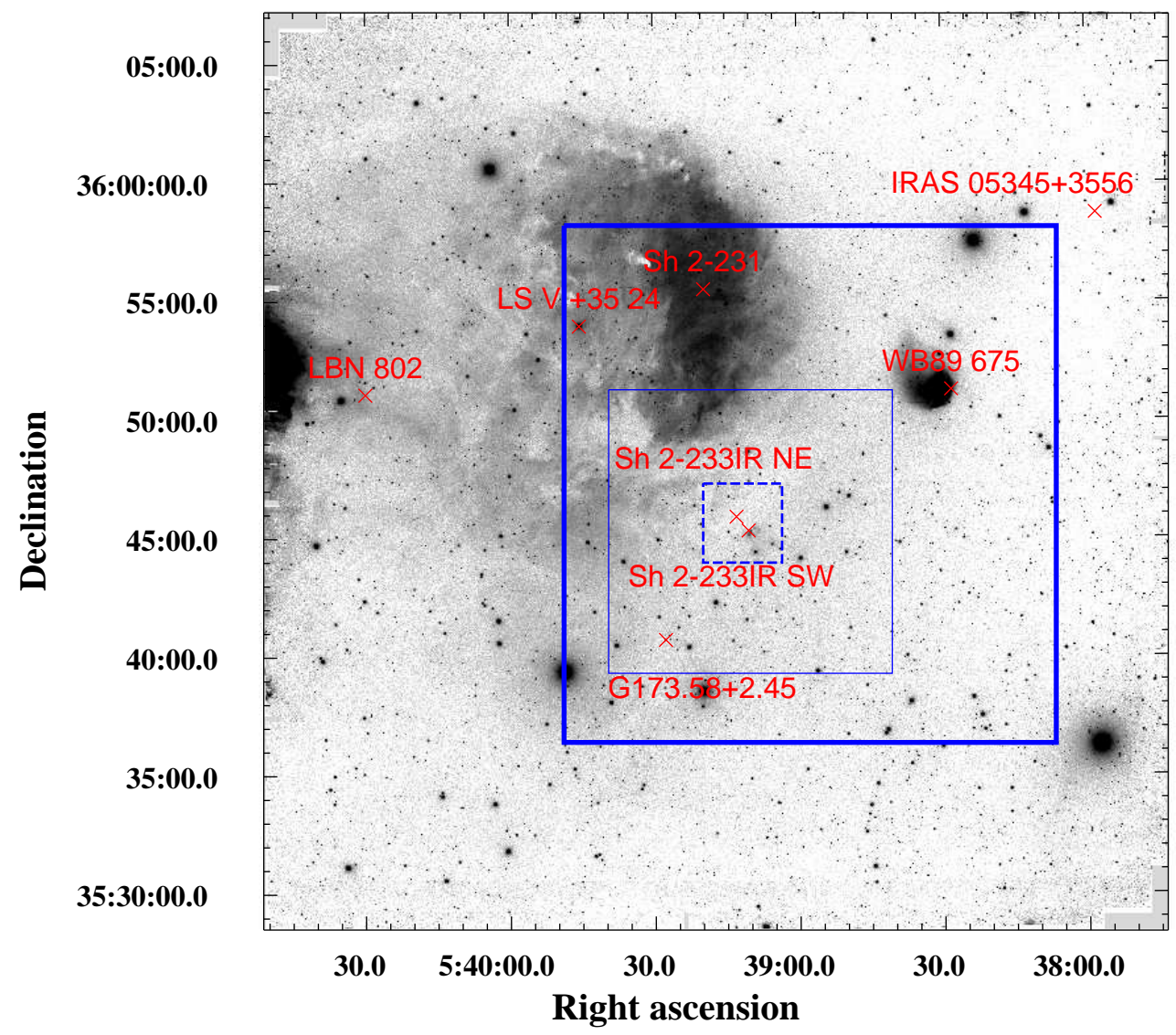

Fig. 1. - The CFHT H $\mathrm{H}_{2}$ (bold), CO 3-2 HARP (thin), and CO 2-1 A3 (dashed) fields overlaid on the KPNO H $\alpha$ mosaic with selected objects identified by their SIMBAD names. Sh 2-233IR SW is coincident with IRAS $05358+3543$. 
used and sensitivities and beam sizes achieved are listed in Tables 1 and 8 . There appeared to be calibration errors in the AR482 observations (the phase calibrator was 2-3 times brighter than in all other observations) and this data were therefore not used in the final analysis, but it produced consistent pointing results.

\section{Results}

\subsection{Near Infrared Imaging: Outflows and Stars}

Eleven distinct outflows have been identified in IRAS 05358 in the images. Outflows are identified from a combination of $\mathrm{J}=3-2 \mathrm{CO}$ data, shock excited $\mathrm{H}_{2}$ emission, and published interferometric maps (Beuther et al. 2002a). Suspected CO outflows were identified by the presence of wings on the $\mathrm{CO} \mathrm{J}=3-2$ emission lines that extended beyond the typical velocity range of emission associated with the line core. The single dish data were compared to the interferometric maps of Beuther et al. (2002a). The CFHT $\mathrm{H}_{2}$ image was then used to search for shock-excited emission associated with the outflow lobes.

Figure 2 shows the $\mathrm{H}_{2} \mathrm{~S}(1)$ 1-0 $2.1218 \mu \mathrm{m}$ (a rovibrational transition in the electronic ground state from the $v=1, J=3$ to the $v=0, J=1$ state) emission in the vicinity of IRAS 05358 with outflows and possible outflow sources labeled. The mm cores from Beuther et al. (2002a) are identified by red squares.

The flow vectors in figure 2 were chosen on the basis of the $\mathrm{H}_{2}$ bow shock morphologies and orientations of chains of $\mathrm{H}_{2}$ features, association with arcsecond-scale $\mathrm{CO}$ features on the Beuther et al. (2002a) Figure 8 CO map, and/or association with lobes of Doppler-shifted CO emission in the CO 3-2 data. The color of the vector indicates the suspected Doppler shift; red and

Table 1. VLA Observation Program Names, Dates, and Times

\begin{tabular}{cccccccc}
\hline \hline $\begin{array}{c}\text { VLA } \\
\text { Observation } \\
\text { Name }\end{array}$ & $\begin{array}{c}\text { Observation } \\
\text { Date }\end{array}$ & $\begin{array}{c}\text { Time } \\
\text { on } \\
\text { Source }\end{array}$ & Array & Band & Fluxcal & Phase cal & $\begin{array}{c}\text { Phase cal } \\
\text { Percent } \\
\text { Uncertainty }\end{array}$ \\
\hline AR482 & August 2 2001 & $2580 \mathrm{~s}$ & $\mathrm{~B}$ & $\mathrm{X}$ & $3 \mathrm{c} 286$ & $0555+398$ & 22 \\
AR513 & June 21 2003 & $7770 \mathrm{~s}$ & $\mathrm{~A}$ & $\mathrm{X}$ & $3 \mathrm{c} 286$ & $0555+398$ & 0.8 \\
AS831 & February 26 2005 & $2640 \mathrm{~s}$ & $\mathrm{~B}$ & $\mathrm{X}$ & $3 \mathrm{c} 286$ & $0555+398$ & 0.7 \\
AS831 & August 5 2005 & $2660 \mathrm{~s}$ & $\mathrm{C}$ & $\mathrm{X}$ & $3 \mathrm{c} 286$ & $0555+398$ & 0.3 \\
AS831 & May 11 2006 & $2610 \mathrm{~s}$ & $\mathrm{~A}$ & $\mathrm{X}$ & $3 \mathrm{c} 286$ & $0555+398$ & 3.0 \\
AL704 & August 7 2007 & $6423 \mathrm{~s}$ & $\mathrm{~A}$ & $\mathrm{Q}$ & $3 \mathrm{c} 273$ & $0555+398$ & 18 \\
AL704 & September 1 2007 & $6423 \mathrm{~s}$ & $\mathrm{~A}$ & $\mathrm{Q}$ & $3 \mathrm{c} 273$ & $0555+398$ & 13 \\
AM697 & November 26 2001 & $2880 \mathrm{~s}$ & $\mathrm{D}$ & $\mathrm{Q}$ & $3 \mathrm{c} 286$ & $0555+398$ & 2.2 \\
AM697 & November 28 2001 & $1530 \mathrm{~s}$ & $\mathrm{D}$ & $\mathrm{K}$ & $3 \mathrm{c} 286$ & $0555+398$ & 2.1 \\
AM697 & November 28 2001 & $1530 \mathrm{~s}$ & $\mathrm{D}$ & $\mathrm{U}$ & $3 \mathrm{c} 286$ & $0555+398$ & 5.8 \\
\hline
\end{tabular}




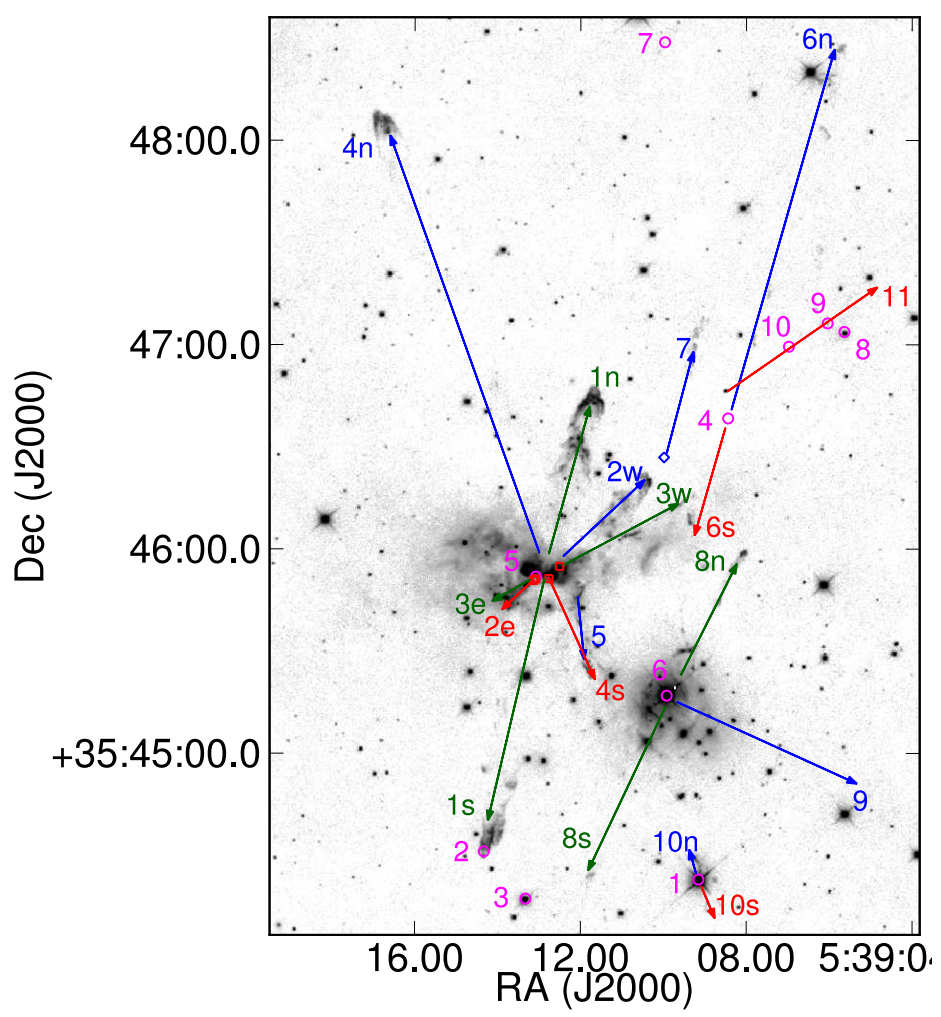

Fig. 2. - The outflows described in section 3.1 overlaid on the $\mathrm{CFHT}_{2}$ image. Numbers followed by $r$ and $b$ (red and blue), $n$ and $s$ (north and south), or $e$ and $w$ (east and west) are thought to be counterflows. Red and blue vectors indicate red and blue doppler shifts. Green vectors indicate where the doppler shift is ambiguous or cannot be determined. Magenta circles are Spitzer $24 \mu \mathrm{m}$ sources. Red squares are Beuther et al. (2002a) mm sources (from left to right, mm1, mm2, mm3). The blue diamond is a YSO candidate detected only in IRAC bands. The length of the vectors corresponds to the approximate length of the outflows. Source 1 and 6 correspond to Porras et al. (2000) IR 6 and IR 41 respectively, and they are discussed under these names in sections 3.1. The bows of Outflow $1 \mathrm{n}$ and $4 \mathrm{n}$ are detected in $\mathrm{H} \alpha$ and [S II] emission and are therefore as identified as Herbig-Haro objects HH 993 and 994 respectively. 


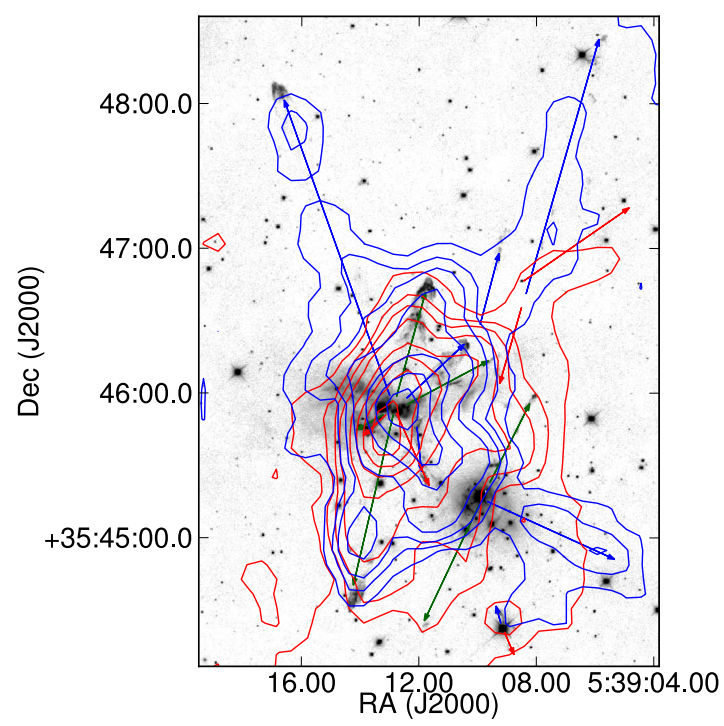

Fig. 3. - CO contours integrated from $v_{L S R}=-13$ to $-4 \mathrm{~km} \mathrm{~s}^{-1}$ (red) and -34 to $-21 \mathrm{~km} \mathrm{~s}^{-1}$ (blue) at levels of $2,4,6,10,20,30,40,50 \mathrm{~K} \mathrm{~km} \mathrm{~s}^{-1}$ overlaid on the $\mathrm{H}_{2}$ image. Specific outflows are labeled in Figure 2 on the same scale.

blue correspond to red and blueshifts and green vectors indicate that the Doppler shift is uncertain.

IRAS 05358 outflow 1: The most prominent flow in $\mathrm{H}_{2}$ is associated with the bright bowshocks N1 and N6 (Khanzadvan et al. 2004) located towards PA $\approx 345^{\circ}$ and $170^{\circ}$ respectively from the sub-mm source mms1b (Beuther et al. 2002a). This flow, Beuther et al. (2002a) outflow A, is associated with redshifted and blueshifted $\mathrm{CO}$ emission. The northern shock is seen in $\mathrm{H} \alpha$ and $[\mathrm{S}$ II] emission (figure 4b) and is given a Herbig-Haro designation HH 993.

This flow is indicated by oppositely directed green vectors from the vicinity of smm1, 2, and 3. It is listed as "Jet 1" in Qiu et al. (2008). Kumar et al. (2002) identified the knot immediately behind the bow shock as a Mach disk. In the Beuther et al. (2002a) interferometric maps, the north flow contains redshifted features and the south flow contains primarily blueshifted features. There are also blueshifted $\mathrm{CO}$ features to the west of the $\mathrm{H}_{2}$ knots that are probably part of a different flow that is not seen in $\mathrm{H}_{2}$ emission.

The velocity of the flow as measured from $\mathrm{H}_{2}$ emssion is blueshifted as much as $80 \mathrm{~km} \mathrm{~s}^{-1}$ (LSR), but one component is blueshifted only $14 \mathrm{~km} \mathrm{~s}^{-1}$ (see table 3), which is consistent with the cloud velocity. A redshifted $\mathrm{SiO}$ lobe is present in the south counterflow. The presence of $\mathrm{H} \alpha$, [S II], and [O III] emission in the north shock and corresponding nondetections in the south shock suggest that there is substantially greater extinction towards the south knot. While the velocities in three of the four apertures picked along the TripleSpec slit are blueshifted, there are also knots with velocities 

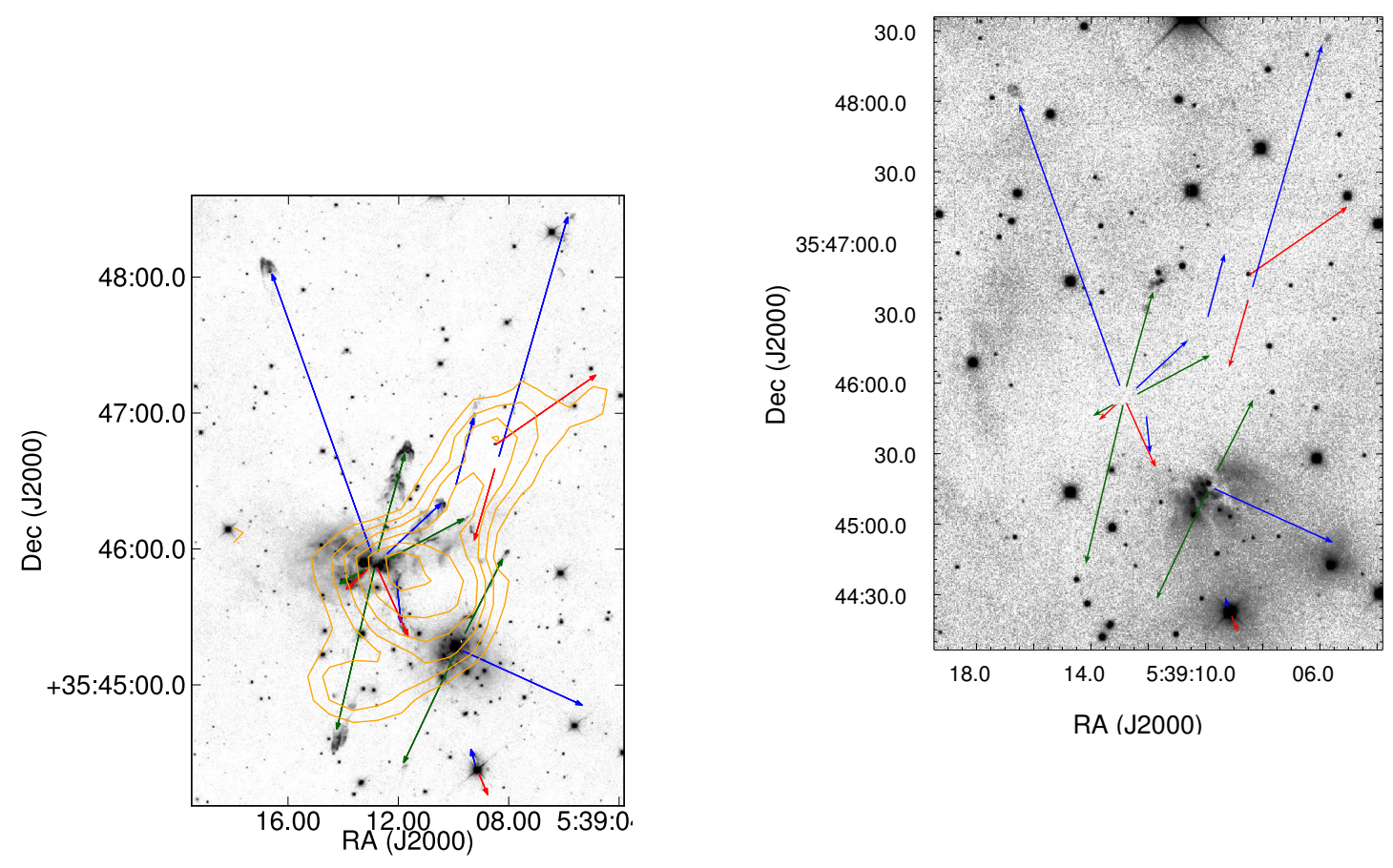

Fig. 4.- (a) $\mathrm{H}_{2}$ image with $\mathrm{SO} 5_{6}-45$ peak flux contours at $0.5-1.4 \mathrm{~K}$ in intervals of $0.15 \mathrm{~K}$ overlaid. With a critical density $\sim 3.5 \times 10^{6}$ (Schöier et al. 2005), this transition is a dense gas tracer. (b) The [S II] image with outflow vectors overlaid. Diffuse emission can be seen at the north ends of Outflows 1, 4, and 6 and around the reflection nebula near source IR 41.

consistent with the cloud velocity. Porras et al. (2000) measure the velocity of the counterflow to be $-17.3 \mathrm{~km} \mathrm{~s}^{-1}$, which is consistent with the cloud velocity. Outflow 1 is propagating very nearly in the plane of the sky.

A line connecting the two bow shocks in Outflow 1 goes directly through Beuther et al. (2007) source mm2a despite the clear association in the Beuther et al. (2002a) interferometric CO map (their Figure 8) with mm1a. The currently available data do not clarify which is the source of the outflow: while the bent $\mathrm{CO}$ outflow appears to trace Outflow 1 back to mm1a, there are additional parallel CO outflows towards the confused central region that could originate from either mm1a or mm2a.

A Spitzer $4.5 \mu \mathrm{m}$ and $24 \mu \mathrm{m}$ source is barely detected in $\mathrm{H}_{2} 2.5^{\prime}$ to the north of Outflow 1 . It is only apparent when the $\mathrm{H}_{2}$ image is smoothed and would have been dismissed as noise except for the association with a probably $4.5 \mu \mathrm{m}$ extended source. It is labeled $24 \mu \mathrm{m}$ source 7 in figure 
2. It appears to be slightly resolved at $4.5 \mu \mathrm{m}$, and is therefore likely shocked emission. The object may be a protostellar source with an associated outflow, but its proximity to the projected path of Outflow 1 suggests that it may be an older outflow knot.

IRAS 05358 Outflow 2: The second brightest $\mathrm{H}_{2}$ features trace a bipolar flow emerging from the immediate vicinity of the sub-mm cluster at $\mathrm{PA} \approx 135^{\circ}$ (red lobe) and $315^{\circ}$ (blue lobe). It is listed as "Jet 2" in Figure 6 of Qiu et al. (2008). The counterflow probably overlaps in the line of sight with the counterflow from Outflow 3 . It is shorter on the counterflow side either because it has already penetrated the cloud and is no longer impacting any ambient gas or, more likely, it has slowly drilled its way out of the molecular cloud and has not been able to propagate as quickly as the northwest flow. The $\mathrm{H}_{2}$ velocities measured for these knots are $\sim 30 \mathrm{~km} \mathrm{~s}^{-1}$ blueshifted, or marginally blue of the cloud LSR velocity.

The disk identified in Minier et al. (2000) is approximately perpendicular to the measured angle of Outflow 2 assuming that mmla is the source of this flow. It is therefore an excellent candidate for the outflow source. A diagram of the mm1a region is shown in figure 13. See Section 3.6 for detailed discussion.

IRAS 05358 outflow 3: The Beuther et al. (2002a) CO and SiO maps reveal a third flow, their outflow $\mathrm{B}$ at $\mathrm{PA} \approx 135^{\circ}$ (red lobe) and $315^{\circ}$ (blue lobe). A chain of $\mathrm{H}_{2}$ features, Khanzadvan et al. (2004) features N3D and N3E, are probably shocks in this flow. It is listed as "Jet 3" in Qiu et al. (2008). The two chains of $\mathrm{H}_{2}$ emission indicate that outflows 2 and 3 are distinct. There also appears to be a counterflow at a shorter distance from the mm cores similar to counterflow 2 .

Outflows 2 and 3 may be associated with either redshifted or blueshifted features in the Beuther et al. (2002a) $\mathrm{CO}$ and $\mathrm{SiO}$ maps. High velocity flows with both parities are present near both the northwest (Beuther et al. (2002a) outflow C) and southeast flow for these jets, but the resolution of the millimeter observations is inadequate to determine which flow is in which direction. Porras et al. (2000) measures $v_{L S R}=-7.5 \mathrm{~km} \mathrm{~s}^{-1}$ for their knot $4 \mathrm{~A}$, which corresponds to the blended southeast counterflow of outflows 2 and 3. Their Figure 7 shows a wide line that is probably better represented by two or three blended lines, one consistent with the cloud velocity and the other(s) redshifted. Since Outflow 2 has a measured blueshift and outflow 3 is significantly fainter, the redshifted counterflow emission is probably associated with Outflow 2 and the blueshifted with outflow 3 .

IRAS 05358 outflow 4: The JCMT CO data and $\mathrm{H}_{2}$ images reveal a large outflow lobe consisting of blue lobes 1 and 4 that form a tongue of blueshifted emission propagating to the northeast at $\mathrm{PA} \approx 20^{\circ}$ (Figure 2) from the cluster of sub-mm cores. A faint chain of $\mathrm{H}_{2}$ features runs along the axis of the $\mathrm{CO}$ tongue and terminates in a bright $\mathrm{H}_{2}$ bow shock located at the northern edge of 2 , Several $\mathrm{H}_{2}$ knots lie along the expected counterflow direction, but that portion of the field contains multiple outflows and is highly confused. If the counterflow is symmetric with the northeast knot, it extends 2.1 parsecs on the sky.

The bow shock of Outflow 4 is seen in the HII and [S II] images, implying that the extinction 
is much lower than in the cluster. Two apertures placed along the bow shock reveal that it is blueshifted about $70 \mathrm{~km} \mathrm{~s}^{-1}$ and may be extincted by as little as $A_{V} \sim .5$. It is designated HH 994 .

IRAS 05358 outflow 5: Figure 2] shows a bright chain of $\mathrm{H}_{2}$ knots and bow shocks starting about $10^{\prime \prime}$ west of $\mathrm{mm} 3$ and propagating south at $\mathrm{PA} \approx 190^{\circ}$. The $\mathrm{SiO}$ maps of Beuther et al. (2002a) show a tongue of blueshifted emission along this chain (their Outflow C). The outflow projects back to $\mathrm{H}^{13} \mathrm{CO}^{+}$source 3 , which is also a weak mm source. A lack of obvious counterflow and the possibility that the knots identified with Outflow 5 could be associated with a number of different crossing flows makes this identification very tentative. Higher spatial resolution observations will be required to determine the association of this outflow.

IRAS 05358 outflow 6: The fourth brightest source in the Spitzer $24 \mu \mathrm{m}$ data is located at $\mathrm{J}(2000)=05: 39: 08.5,+35: 46: 38$ (source 5 in the IRAS 05358 section of the Qiu et al. (2008) catalog, referred to in table 3 as Q5) in the middle of the molecular ridge that extends from IRAS 05358 towards the northwest $(24 \mu \mathrm{m}$ object 4 in figure 21). The star is located at the northwest end of the tongue of $1.2 \mathrm{~mm}$ emission mapped by (Beuther et al. 2002a) with the MAMBO instrument on the IRAM telescope. This part of the cloud is also seen in silhouette against brighter surrounding emission at $8 \mu \mathrm{m}$. At wavelengths below $2 \mu \mathrm{m}$, it is fainter than 14-th magnitude and therefore is not listed in the 2MASS catalog, and it is not detected in Yan (2009) down to 19th magnitude in K.

Spitzer data indicates very red colors between 3.6 and $70 \mu \mathrm{m}$, indicating that this object is likely to be a Class I protostar. The SED is fit using the online tool provided by Robitaille et al. (2007). Unfortunately, a wide variety of parameters all achieved equally good fits, so no conclusions are drawn about the stellar mass or other very uncertain parameters. However, the top models all had $A_{V}>20$ and many in the range 30-50, indicating that the line of sight is probably through a thick envelope or disk towards this source.

This source lies at the base of the tongue of blueshifted CO 3-2 emission that extends northwest of IRAS 05358 at $\mathrm{PA} \approx 345^{\circ}$ and has mass $\sim .5 M_{\odot}$. A pair of $\mathrm{H}_{2}$ features, Khanzadvan et al. (2004) $\mathrm{N} 12 \mathrm{~A}$ and $\mathrm{N} 12 \mathrm{~B}$ are located 30 and $55^{\prime \prime}$ from the suspected YSO, forming a chain along the axis of the blueshifted CO tongue. Khanzadyan et al. (2004) $\mathrm{H}_{2}$ knot N3F lies along the flow axis in the redshifted direction.

IRAS 05358 outflow 7: The 20" long chain of $\mathrm{H}_{2}$ knots labeled Khanzadyan et al. (2004) N11 appears to trace part of a jet at $\mathrm{PA} \approx 345^{\circ}$ that propagated parallel to outflow 6 about $20^{\prime \prime}$ to the east. The northwest portion of Outflow $\mathrm{C}$ in the Beuther et al. (2002a) SiO map is in approximately the same direction as Outflow 7, and it may represent a redshifted counterflow to the northwestpointing $\mathrm{H}_{2}$ knots. The jet axis passes within a few arc-seconds of a faint and red YSO located at $\mathrm{J}(2000)=053910.0,+354627$ (blue diamond in figure 2 about 35" south of the southern end of the $\mathrm{H}_{2}$ feature). It may be a $24 \mu \mathrm{m}$ source but is lost in the PSF of the bright source at the center of Sh 2-233IR NE. This object is also undetected down to 19th magnitude in the Yan (2009) K-band image. 
IRAS 05358 outflow 8: A prominent jet-like $\mathrm{H}_{2}$ feature protrudes from the vicinity of Sh 2233IR SW at $\mathrm{PA} \approx 335^{\circ}$ and ends in bright knot N9. The feature N5B is is located just outside the ring of $\mathrm{H}_{2}$ emission that surrounds the IRAS source at the base of the jet. Towards the southeast, knot N6 is located opposite knot N9 with respect to the southwest cluster. IR 41, the H $\alpha$ emission source, labeled $24 \mu \mathrm{m}$ source 6 in figure 2, is probably the source of this outflow.

IRAS 05358 outflow 9: In the Spitzer and $\mathrm{K}_{s}$ images, an infrared reflection nebula opens towards the southwest at $\mathrm{PA} \approx 245^{\circ}$ and points towards a blueshifted $\mathrm{CO}$ region. The reflection nebula is also seen in $\mathrm{H} \alpha$. It is likely that the CO emission in CO Region 1 (table 2) traces a fossil cavity whose walls provide the scattering surface of the reflection nebula.

IRAS 05358 outflow 10 and IR 6: A bright $\mathrm{H}_{2}$ filament protrudes at $\mathrm{PA} \approx 15^{\circ}$ towards the northeast of IR $6(24 \mu \mathrm{m}$ source 1, Qiu et al. (2008) source 8). The star is the third brightest $24 \mu \mathrm{m}$ source in the IRAS 05358 region. Since it is visible at visual wavelengths, it is not heavily embedded. Its $\mathrm{H} \alpha$ emission and association with an outflow lobe and $\mathrm{H}_{2}$ emission suggest that it is a moderate mass Herbig AeBe star associated with the IRAS 05358 complex. The optical spectrum confirms this hypothesis: the star has $\mathrm{H} \alpha$ absorption wings on either side of a very bright, asymmetric $\mathrm{H} \alpha$ emission profile (see section 3.5).

IR 6 is seen to be the source of Outflow 10. Data for this source is available from $\sim 0.45-$ $24 \mu \mathrm{m}$, so the Robitaille et al. (2007) spectral fitter puts strong constraints on the star's mass and luminosity. The measured mass and luminosity are $M=4.5 \pm 0.5 M_{\odot}$ and $L=10^{2.3 \pm .25} L_{\odot}$, parameters consistent with a $\mathrm{B} 7 \mathrm{~V}( \pm 1$ spectral class $)$ main sequence star. The range of ages in the models covers $10^{4}-10^{7}$ years but favors stars in the range $10^{5}-10^{6}$ years.

While there is a small clump of redshifted $\mathrm{CO}$ emission to the northeast of the object, the $\mathrm{H}_{2}$ spectrum shows that the north flow is blueshifted $v_{L S R} \sim-40 \mathrm{~km} \mathrm{~s}^{-1}$, and the lack of a visible counterflow suggests that the counterflow may be masked behind an additional extincting medium. The counterflow drawn in figure 2 is not seen in emission but is identified as a probable location for a counterflow because of the confident association of outflow 10n with source IR 6 .

IRAS 05358 outflow 11: A chain of $\mathrm{H}_{2}$ knots is seen at $2.12 \mu \mathrm{m}$ and in the Spitzer $4.5 \mu \mathrm{m}$ image. They trace back to either IR 78 or $24 \mu \mathrm{m}$ source 4 . There is a tongue of redshifted CO 3-2 emission in the same direction as this flow that suggests it may be redshifted.

IR 41: There is an arc-like $\mathrm{H}_{2}$ emission feature surrounding the $\mathrm{H} \alpha$ emission line star IR 41. This implies that the star is probably a late B-type star with too little Lyman continuum emission to generate a photon-dominated region (PDR) but enough soft UV to excite $\mathrm{H}_{2}$. From the measured $\mathrm{H} \alpha$ and nondetection of $\mathrm{H} \beta$ at the star's location down to a $5-\sigma$ limit of $1 \times 10^{-17}$ $\operatorname{erg~s}^{-1} \mathrm{~cm}^{-2} \AA^{-1}$, a lower limit on the extinction column $A_{V}=15$ is derived. The Robitaille et al. (2007) fitter yields a mass estimate of $7.4 \pm 0.6 M_{\odot}$ and luminosity $L=10^{2.97 \pm .16} L_{\odot}$ among the 222 best fits out of a grid of 200,000 model SEDs (fits with $\chi^{2}<5000$ ). The luminosity is very well constrained, varying only modestly to $L=10^{2.99 \pm .15} L_{\odot}$ for the 904 best fits $\left(\chi^{2}<10000\right)$, while the mass shifts down to $6.5 \pm 1.0 M_{\odot}$. The mass estimate may be biased by the lower number of 
high-mass models computed. The star's mass is most compatible with a main sequence B4V star, though its luminosity is closer to a B5V star. The disk mass is constrained to be $>10^{3} M_{\odot}$. The age is reasonably well constrained to be $T=10^{5.78 \pm .12}$ for the best 904 models, but is essentially unconstrained for the best 222. Similarly, the stellar temperature is entirely unconstrained by the fitting process.

The very high values of $\chi^{2}$ would normally be worrisome, but the $\chi^{2}$ statistic only represents statistical error, while the data is dominated by various systematic errors including calibration offsets in the optical/NIR and poor resolution in the far-IR. Therefore, it is not possible to find a perfect model fit, but still possible to put constraints on the physical properties of the source.

South of IRAS 05358: There is a symmetric flow with one faint $\mathrm{H}_{2}$ knot and a bright central source about $4^{\prime}$ south of IRAS 05358. The $\mathrm{H}_{2}$ knot is at $\mathrm{J}(2000)=05: 39: 15.63+35: 42: 13.2$. The flow has a clear red and blue region as identified in figure 6, the red flow extends from -9 to -14 $\mathrm{km} \mathrm{s}^{-1}$ and the blue from -19 to $-23 \mathrm{~km} \mathrm{~s}^{-1}$ (the outflow is swamped by ambient emission in the intermediate velocity range). The outflow is $\sim 2^{\prime}$ long, though the probable source identified is not directly between the two lobes. The ellipses used are labeled in table 2 as Red S and Blue S.

\subsection{Imaging results: Optical}

Deep [S II] images show that some of the outflows have pierced through the obscuring dust layers or excited extremely bright sulfur emission. Khanzadyan et al. (2004) knot N1 at the end of Outflow 1 is visible [S II] emission The bow of outflow 4 and the northwest end of outflow 6 are detected in [S II]. Only the Outflow 1 and 4 bows are detected in $\mathrm{H} \alpha$ emission, indicating that the emission is most likely from shock heating, not external photoionizing radiation. If the shocks were externally irradiated, we would expect the emission to be dominated by the recombination lines. Because they have been detected in the optical, these two flows can be classified as Herbig-Haro objects.

\subsection{CO results}

IRAS 05358 is located at the center of the CO 3-2 integrated velocity maps (Figure 66). The parent molecular cloud, centered at $v_{L S R}=-17.5 \mathrm{~km} \mathrm{~s}^{-1}$, extends from the southeast towards the northwest with the brightest emission coming from the core associated with Sh 2-233IR SW, while the highest integrated emission is associated with Sh 2-233IR NE. Sh 2-233IR NE has a central velocity of $\sim-16.0 \mathrm{~km} \mathrm{~s}^{-1}$ from the optically thin $\mathrm{C}^{18} \mathrm{O} 2-1$ measurements. Material that has been swept up and accelerated by jets and outflows can be seen at velocities $v_{L S R}<-21$ $\mathrm{km} \mathrm{s}^{-1}$ and $v_{L S R}>-12 \mathrm{~km} \mathrm{~s}^{-1}$ (Figure 6). The integrated CO 3-2 map peaks at $\mathrm{J}(2000)=$ 05:39:12.8 $+35: 45: 55$, while the highest observed brightness temperature is at $\mathrm{J}(2000)=5: 39: 09.4$ $+35: 45: 12$. This offset is discussed in the context of CO isotopologues in section 4.4.2 and in section 


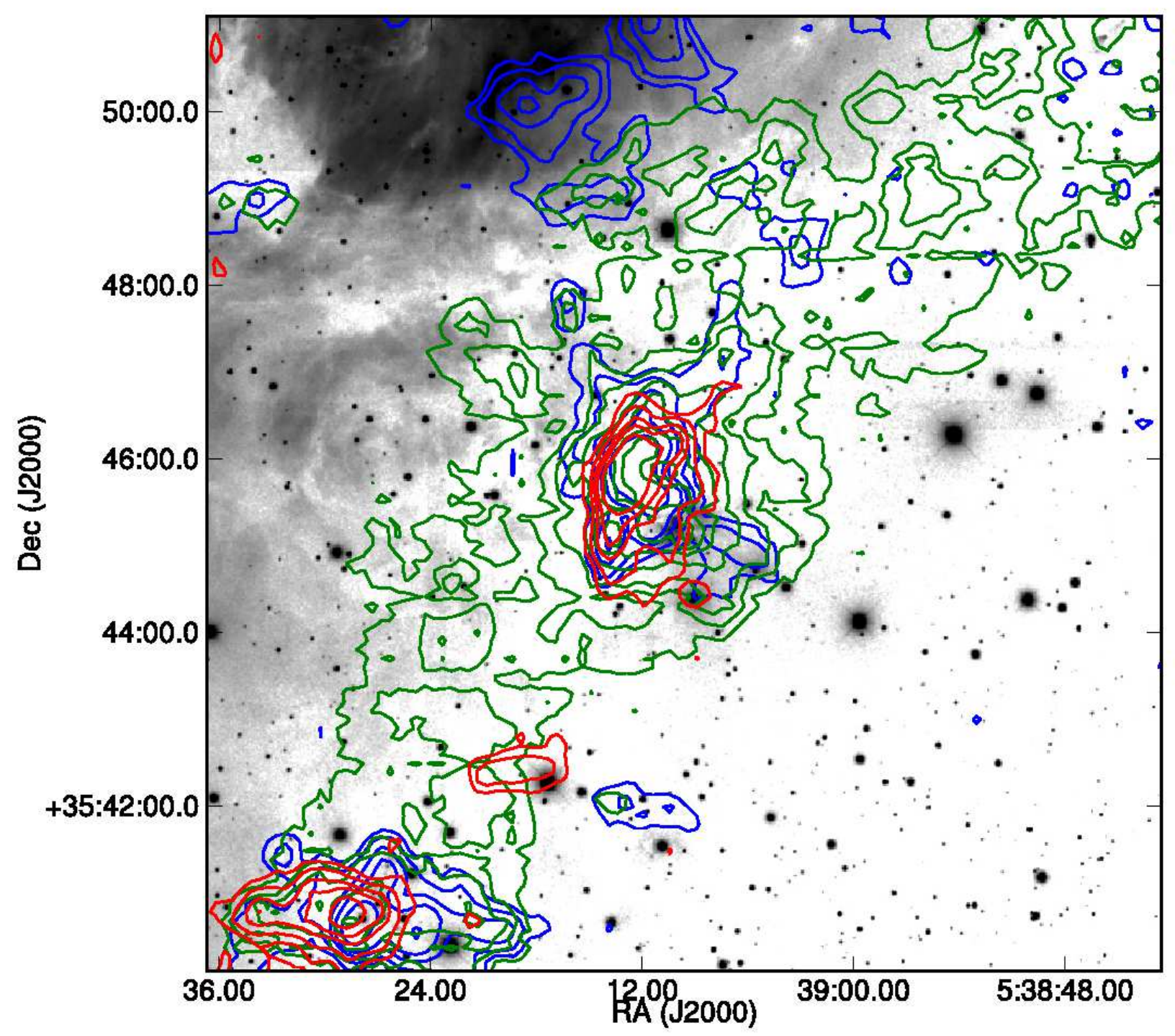

Fig. 5.- The $\mathrm{H} \alpha$ image with $\mathrm{CO}$ contours at redshifted, blueshifted, and middle velocities in red, blue, and green respectively. Contours are at 2,4,8,12,20 $\mathrm{K} \mathrm{km} \mathrm{s}^{-1}$ for the red and blue, and $20,25,30,40,50,60,70 \mathrm{~K} \mathrm{~km} \mathrm{~s}^{-1}$ for the green. Red is integrated from -12 to $-4 \mathrm{~km} \mathrm{~s}^{-1}$, Blue from -31 to $-21 \mathrm{~km} \mathrm{~s}^{-1}$, and green from -21 to $-12 \mathrm{~km} \mathrm{~s}^{-1}$. 
5.1 .

Regions with line wings relative to the ambient cloud within $5^{\prime}$ of the northeast cluster were assumed to be associated with outflows from the cluster. Further than $5^{\prime}$, it is likely that the high velocity wings are accelerated by neighboring HII regions (see section 5.4). These line wings were integrated over the velocity range -34 to $-21 \mathrm{~km} \mathrm{~s}^{-1}$ (blue) and -12 to $1 \mathrm{~km} \mathrm{~s}^{-1}$ (red) to acquire estimates of the outflowing mass under the assumption that outflowing gas is optically thin. The extracted regions are displayed in Figure 6 $\mathrm{b}$ and measurements in table 2 . The line wings in the central arcminute and central 5 arcminutes were measured for comparison with lower resolution data and to compute a total outflow mass in the central region.

The objects in Table 2 labeled CO Region 1, 2, and 3 have uncertain associations with outflows. CO Region 1 is tentatively associated with outflow 11. CO region 2 may be associated with Outflow 3 but is in a highly confused region and may have many contributors. CO region 3 is probably associated with outflow 10. In contrast, the associations with outflows 4 and 6/7 are more certain because they are further from the central region and less confused. Outflow 1 is seen at high velocities in Beuther et al. (2002a) interferometer maps. Outflow 9 is selected primarily based on CO emission.

\subsection{Near-infrared spectroscopy: Velocities}

The slit positions used and apertures extracted from those slits are displayed in Figure 10. Position-velocity diagrams of the 1-0 S(1) line are displayed in Figure 11, Velocity measurements are presented in Table 3 ,

The near-IR spectrum of Outflow 1 has the largest signal. All of the K-band $\mathrm{H}_{2}$ lines except the 2-1 S(0) $2.3556 \mu \mathrm{m}$ (too weak) and 1-0 S(4) $1.8920 \mu \mathrm{m}$ (poor atmospheric transmission) lines were detected (see Table 5). Velocities from gaussian fits to each line are reported. In the central portion of Sh 2-233IR NE, outflowing $\mathrm{H}_{2}$ emission at $v_{L S R} \approx-30 \mathrm{~km} \mathrm{~s}^{-1}$ is detected. This material may be associated with a line-of-sight flow, or may originate from the base of the already identified flows 1-3. In source IR 58, $\operatorname{Br} \gamma$ and $\mathrm{He}$ I 2.05835 $\mu m$ are detected, indicating that there is an embedded PDR in this source. There is a hint of a second, fainter star adjacent to IR 58. IR 93 is observed to be a double source in the TripleSpec spectrum, but the spectrum is too weak to do any identification. Br $\gamma$ and possibly He I are detected at fainter levels.

Table 5 shows the measured line strengths (when detected) of all $\mathrm{H}_{2}$ lines in each aperture. The errors listed are statistical errors that do not include the systematics errors introduced by a failure to correct for narrow atmospheric absorption lines. 


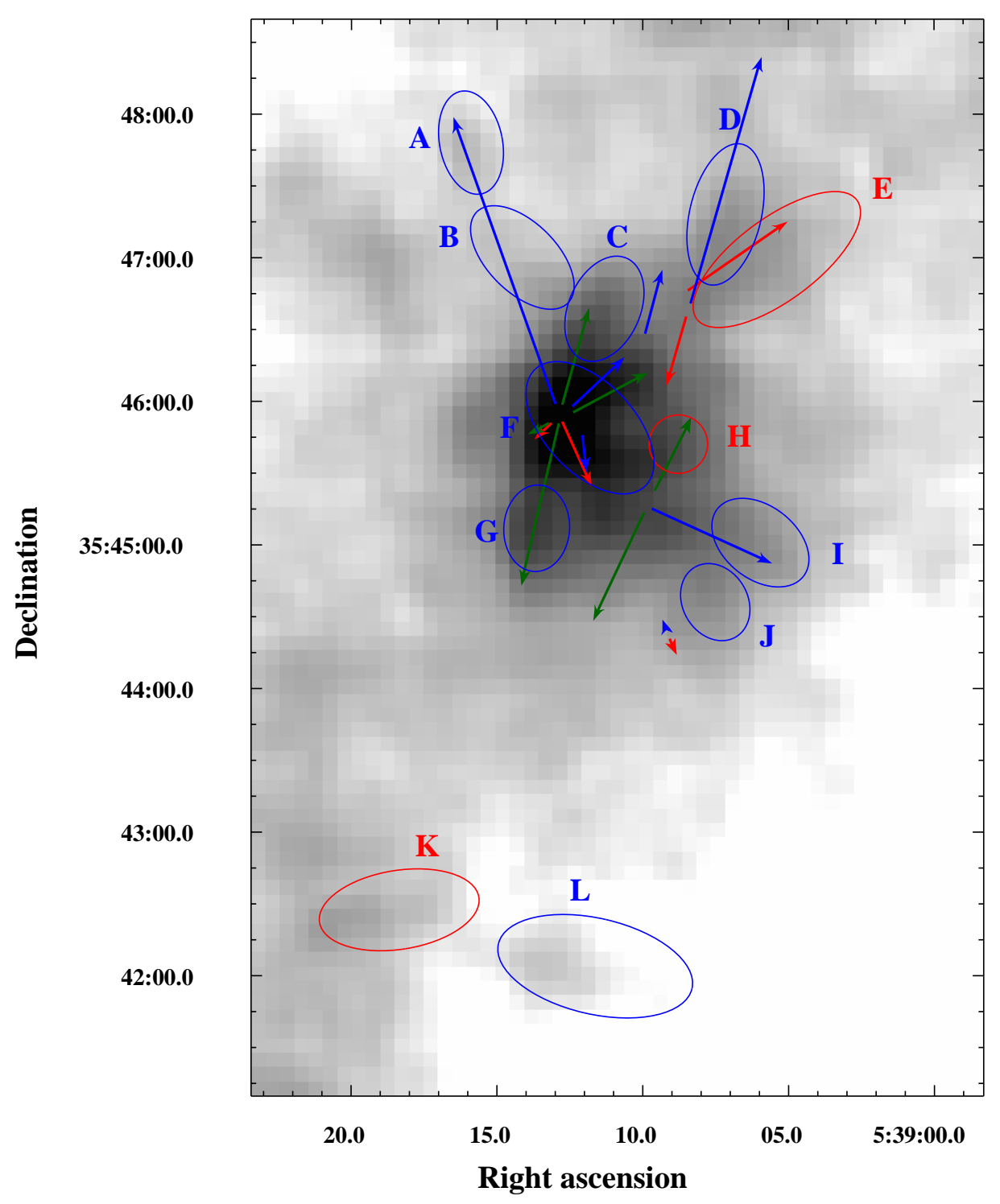

Fig. 6. - The JCMT HARP CO J=3-2 map integrated over all velocities with significant emission $\left(-34 \mathrm{~km} \mathrm{~s}^{-1}\right.$ to $\left.-4 \mathrm{~km} \mathrm{~s}^{-1}\right)$ shown in gray log scale from 0 to $150 \mathrm{~K} \mathrm{~km} \mathrm{~s}^{-1}$. The elliptical regions over which line wings were integrated are shown with blue and red circles corresponding to blue and red line wings. The measurements are presented in table 2 . 


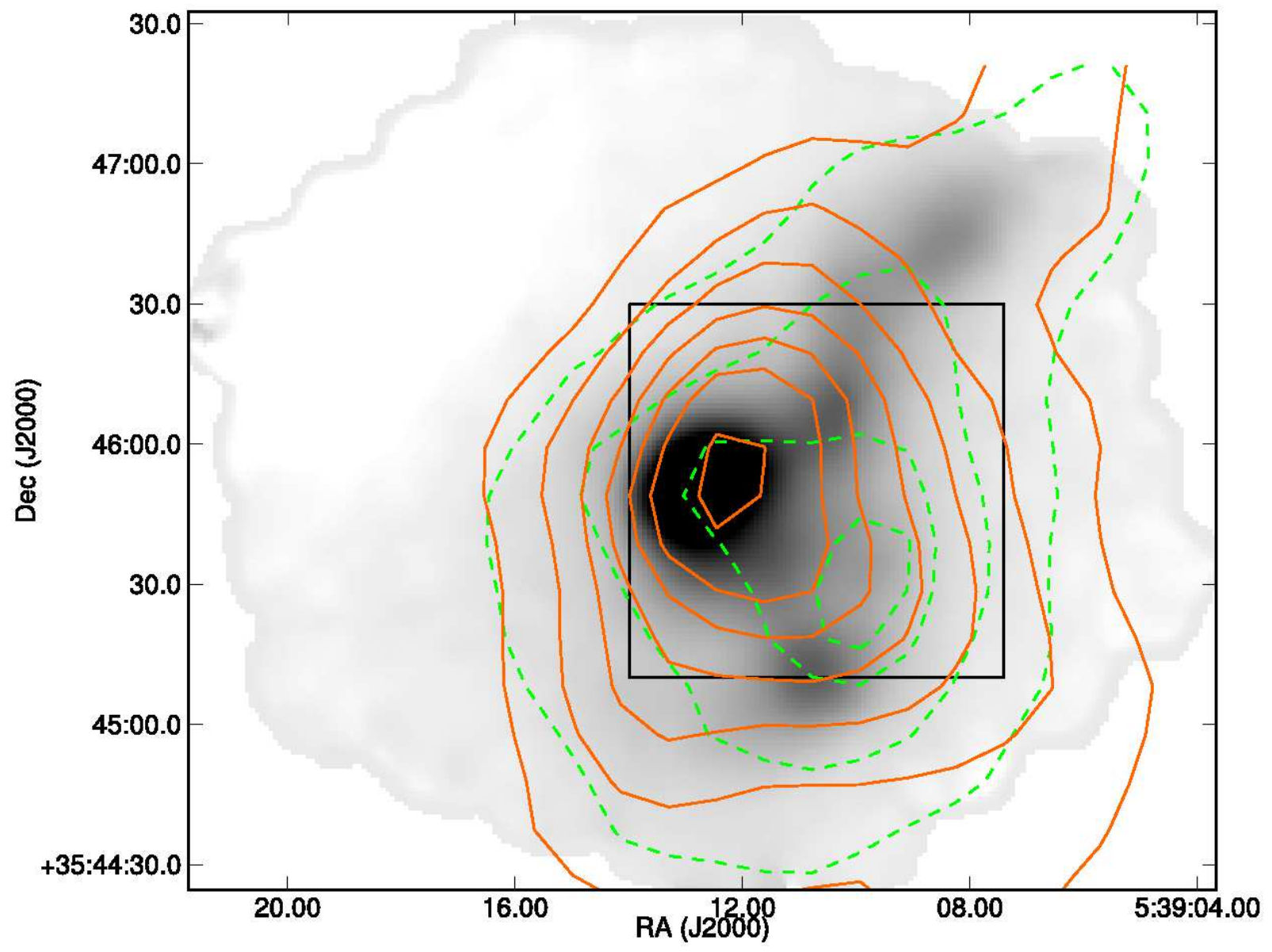

Fig. 7.- SCUBA $850 \mu \mathrm{m}$ image in linear grayscale from -1 to $+10 \mathrm{mJy} / \mathrm{beam}$, with a saturated peak of $24 \mathrm{mJy} /$ beam, with ${ }^{12} \mathrm{CO} 2-1$ (orange solid, contours at 45,60,85,100,115,130,145 $\mathrm{K} \mathrm{km} \mathrm{s}^{-1}$ ) and ${ }^{13} \mathrm{CO} 2-1$ (green dashed, contours at 20,30,40,45 $\mathrm{K} \mathrm{km} \mathrm{s}^{-1}$ ) integrated contours. The box shows the region plotted in Figure 8 . 


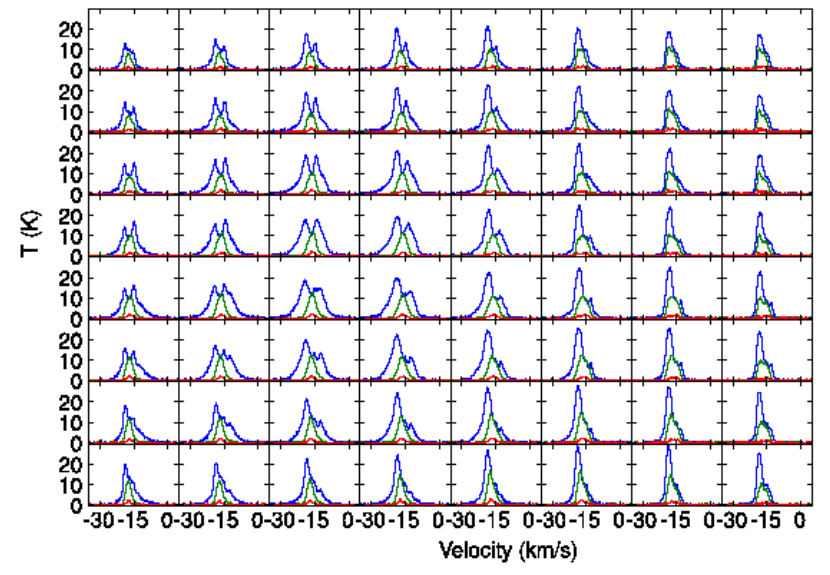

Fig. 8.- CO spectra of Sh 2-233IR NE in ${ }^{12} \mathrm{CO}$ (blue), ${ }^{13} \mathrm{CO}$ (green), and $\mathrm{C}^{18} \mathrm{O}$ (red). The top-left plot is the pixel centered at $\mathrm{J}(2000)=5: 39: 13.67+35: 46: 26.0$ and each pixel is $10^{\prime \prime}$ on a side. The region mapped here is shown with a box in Figure 7. Redshifted self-absorption, a possible infall tracer, is evident in the ${ }^{12} \mathrm{CO}$ spectra in the outer pixels. The inner pixels show self-absorption only at central velocities: this may be an indication that emission from outflows dominates any infall signature, or simply that there is no bulk infall towards Sh 2-233IR NE. 
Table 2. Measured properties of CO flows

\begin{tabular}{|c|c|c|c|c|c|}
\hline${ }^{\mathrm{a}}$ Region Name & $\int T_{m b} *$ & $M\left(M_{\odot}\right)$ & $\mathrm{p}\left(M_{\odot} \mathrm{km} \mathrm{s}^{-1}\right)$ & $\mathrm{N}\left(\mathrm{cm}^{-2}\right)$ & $\mathrm{E}\left(10^{42} \mathrm{erg}\right)$ \\
\hline${ }^{\mathrm{b}}$ A. Outflow $4 \mathrm{a}$ & 4.27 & .022 & .15 & $1.4 \times 10^{19}$ & 11 \\
\hline b B. Outflow 4b & 4.60 & .032 & .21 & $1.5 \times 10^{19}$ & 13 \\
\hline${ }^{\mathrm{b}}$ C. Outflow $1 \mathrm{n}$ & 14.5 & .088 & .71 & $4.8 \times 10^{19}$ & 66 \\
\hline b D. Outflow 6/7 & 4.45 & .045 & .30 & $1.5 \times 10^{19}$ & 29 \\
\hline${ }^{\mathrm{r}}$ E. CO Region 3 & 1.31 & .016 & .112 & $4.3 \times 10^{18}$ & 8.5 \\
\hline b F. Sh 2-233IR NE & 41.8 & .464 & 3.72 & $1.4 \times 10^{20}$ & 330 \\
\hline${ }^{\mathrm{m}}$ F. Sh 2 -233IR NE & 132.9 & 1.47 & - & $4.4 \times 10^{20}$ & - \\
\hline${ }^{\mathrm{r}}$ F. Sh 2-233IR NE & 30.0 & .333 & 2.03 & $9.9 \times 10^{19}$ & 135 \\
\hline${ }^{\mathrm{b}}$ G. Outflow1s & 14.6 & .064 & .48 & $4.8 \times 10^{19}$ & 40 \\
\hline${ }^{\mathrm{r}}$ H. CO Region 2 & 4.54 & .012 & .074 & $1.5 \times 10^{19}$ & 5 \\
\hline b I. Outflow 9 & 6.33 & .039 & .39 & $2.1 \times 10^{19}$ & 43 \\
\hline b J. CO Region 1 & 3.61 & .015 & .12 & $1.2 \times 10^{19}$ & 11 \\
\hline${ }^{\mathrm{r}} \mathrm{K}$. Red S & 5.26 & .051 & .34 & $1.7 \times 10^{19}$ & 26 \\
\hline${ }^{\mathrm{b}}$ L. Blue $\mathrm{S}$ & 3.66 & .053 & .47 & $1.2 \times 10^{19}$ & 47 \\
\hline b $1^{\prime}$ aperture ${ }^{\mathrm{c}}$ & 15.1 & .96 & 7.6 & $5.0 \times 10^{19}$ & 670 \\
\hline b $3^{\prime}$ aperture & 2.7 & 1.6 & 12 & $9.0 \times 10^{18}$ & 1000 \\
\hline${ }^{\mathrm{b}} 5^{\prime}$ aperture & 1.7 & 2.7 & 20 & $5.6 \times 10^{18}$ & 1600 \\
\hline r $1^{\prime}$ aperture & 11.8 & 0.75 & 4.7 & $3.9 \times 10^{19}$ & 320 \\
\hline${ }^{\mathrm{r}} 3^{\prime}$ aperture & 1.9 & 1.1 & 6.8 & $6.2 \times 10^{18}$ & 460 \\
\hline${ }^{\mathrm{r}} 5^{\prime}$ aperture & 0.96 & 1.5 & 10 & $3.2 \times 10^{18}$ & 640 \\
\hline b $1^{\prime}{ }^{12} \mathrm{CO} 2-1$ & 10.4 & .94 & 7.1 & $4.9 \times 10^{19}$ & 590 \\
\hline${ }^{\mathrm{m}} 1^{\prime}{ }^{12} \mathrm{CO} 2-1$ & 97.78 & 8.83 & - & $4.6 \times 10^{20}$ & - \\
\hline${ }^{\mathrm{r}} 1^{\prime}{ }^{12} \mathrm{CO} 2-1$ & 9.17 & 0.83 & 5.52 & $4.3 \times 10^{19}$ & 430 \\
\hline${ }^{\mathrm{m}} 1^{\prime}{ }^{13} \mathrm{CO} 2-1$ & 41.12 & 211 & - & $1.1 \times 10^{22}$ & - \\
\hline${ }^{\mathrm{m}} 1^{\prime} \mathrm{C}^{18} \mathrm{O} 2-1$ & 5.31 & 271 & - & $1.4 \times 10^{22}$ & - \\
\hline
\end{tabular}

${ }^{a}$ Unless labeled otherwise, regions are extracted from CO 3-2 data as shown in figure 6b

${ }^{\mathrm{b}}$ Blue integration over velocity range -34 to $-21 \mathrm{~km} \mathrm{~s}^{-1}$

${ }^{\mathrm{c}}$ Apertures are centered on $\mathrm{J}(2000)=05: 39: 11.238+35: 45: 41.80$ in Sh 2-233IR NE

${ }^{\mathrm{r}}$ Red integration over velocity range -13 to $-4 \mathrm{~km} \mathrm{~s}^{-1}$

${ }^{\mathrm{m}}$ Middle range integration over $-21 \mathrm{~km} \mathrm{~s}^{-1}$ to $-13 \mathrm{~km} \mathrm{~s}^{-1}$. Assumed not to be outflowing, so no momentum is computed 


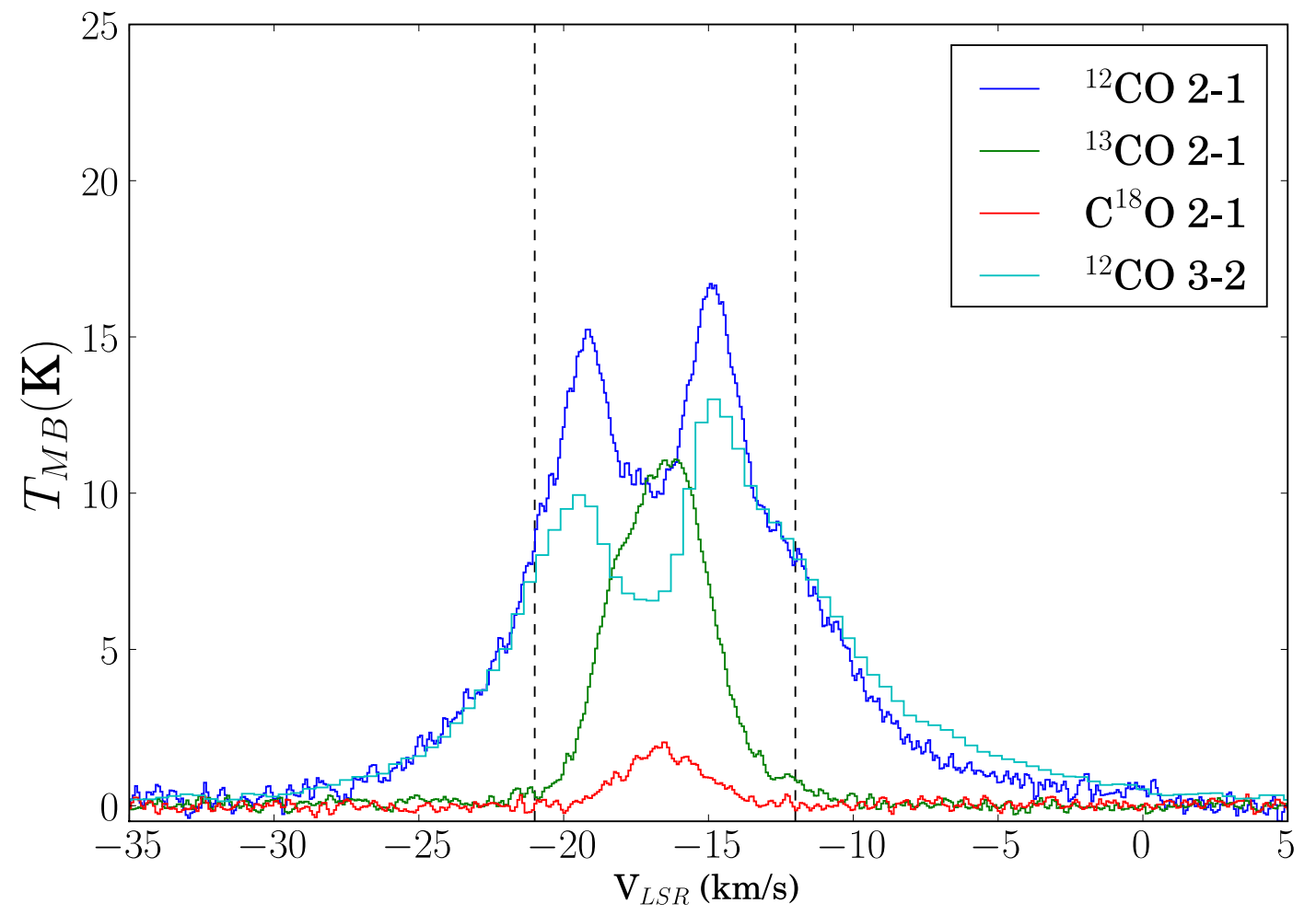

Fig. 9. - CO spectra of inner $12^{\prime \prime}$ centered on Sh 2-233IR NE for all observed CO lines. The CO 3-2 and 2-1 beams are not matched, but in both cases the area integrated over is 1-2 resolution elements across. The divisions demarcating the red and blue line wings are shown with vertical dashed lines at $v_{L S R}=-21$ and $-12 \mathrm{~km} \mathrm{~s}^{-1}$. 


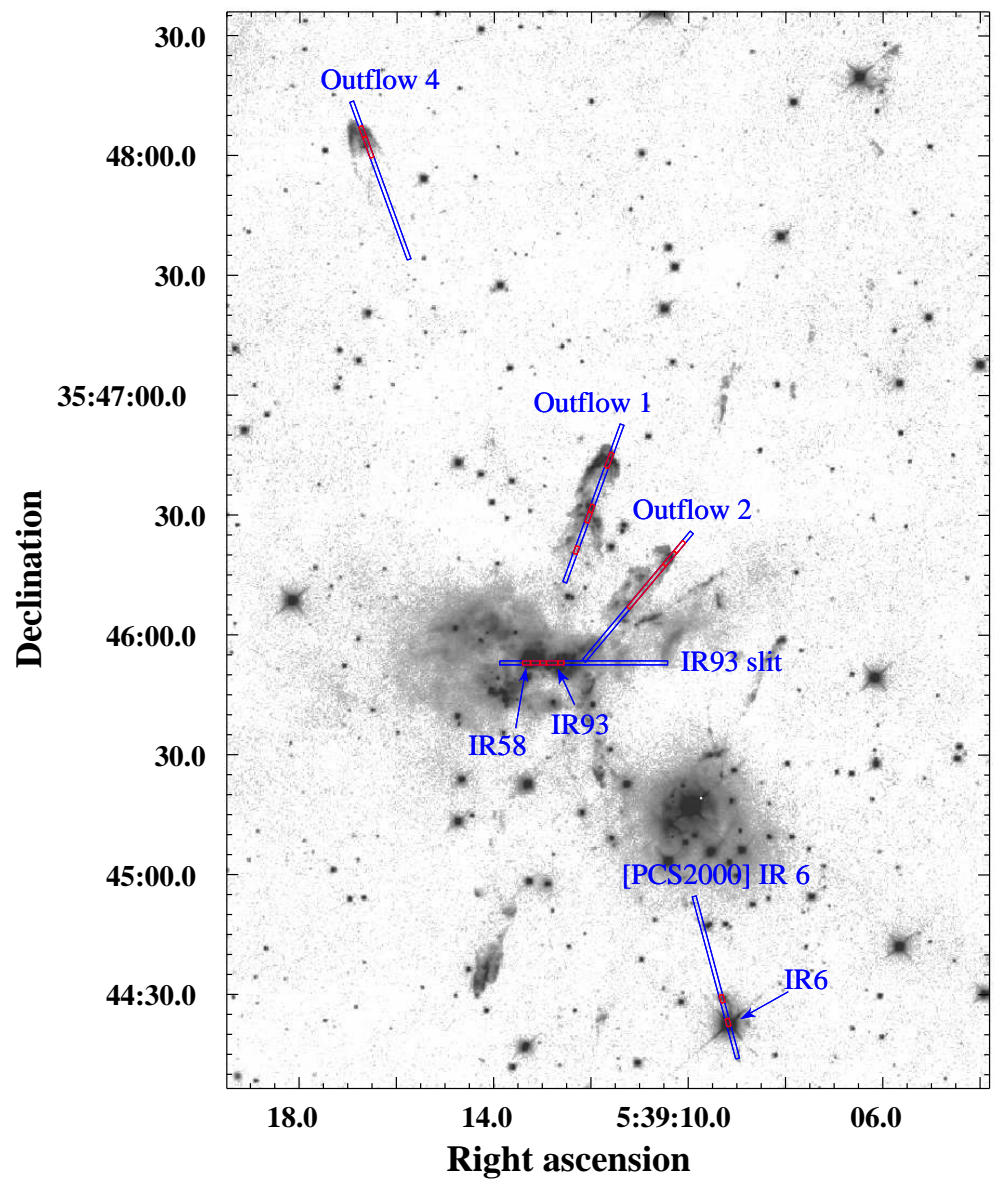

Fig. 10. - TripleSpec slits (blue) overlaid on the $\mathrm{H}_{2}$ image. The red boxes indicate the apertures extracted from those slits to fit and measure $\mathrm{H}_{2}$ properties. The apertures are also indicated in the position-velocity diagrams. 

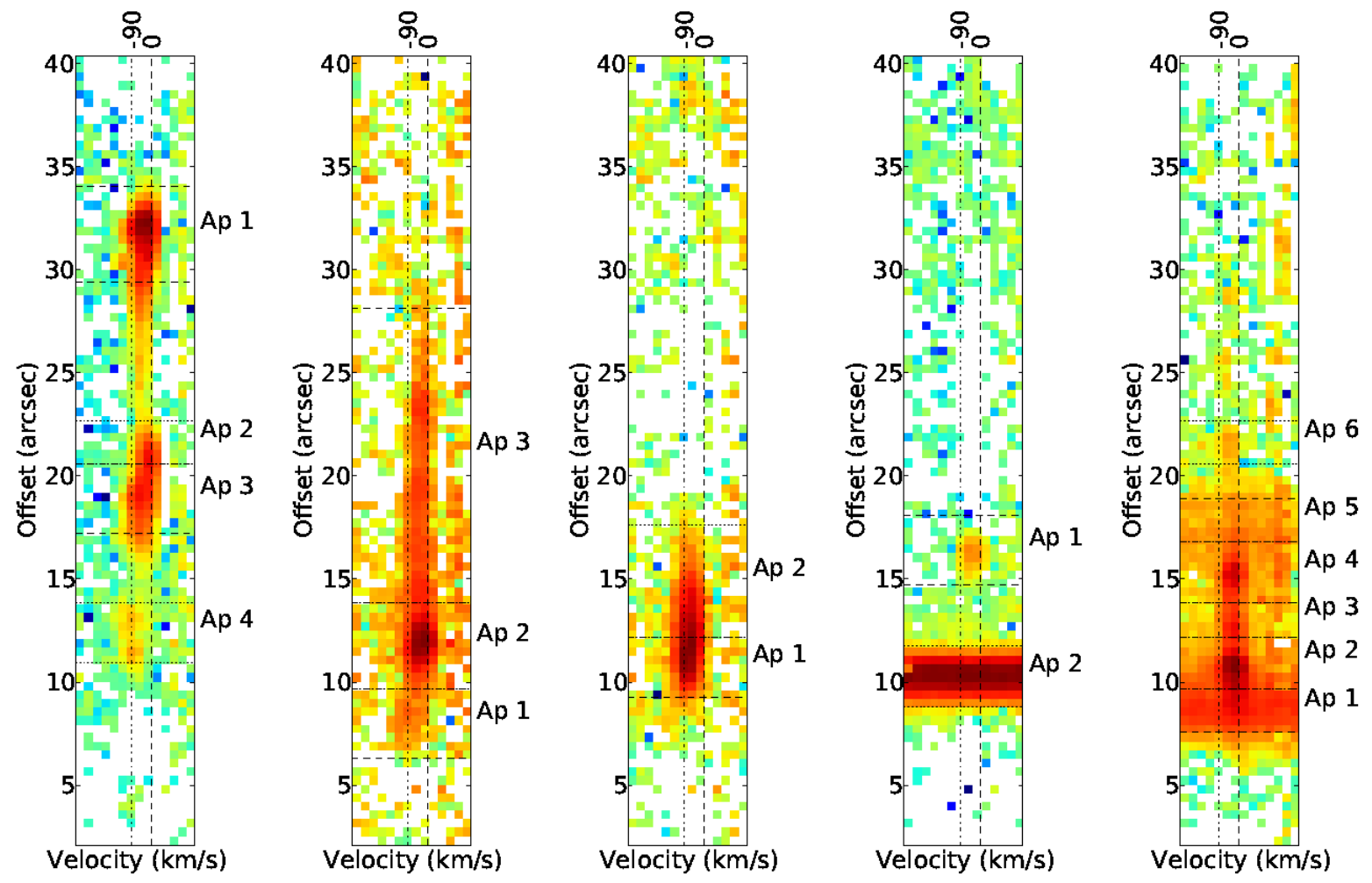

Fig. 11. - Position-velocity diagrams of the $\mathrm{H}_{2} 2.1218 \mu \mathrm{m}$ line in Outflows 1,2, 4, IR 6, and IR93/IR58. The velocity range is from -340 to $190 \mathrm{~km} \mathrm{~s}^{-1}$.

Table 3. TripleSpec fitted $\mathrm{H}_{2}$ outflow velocities

\begin{tabular}{cccc}
\hline \hline Outflow Number & Aperture Number & ${ }^{\mathrm{a}} \mathrm{v}_{L S R}\left(\mathrm{~km} \mathrm{~s}^{-1}\right)$ & $\mathrm{b}_{L S R}\left(\mathrm{~km} \mathrm{~s}^{-1}\right)$ \\
\hline 1 & 1 & $-33.54(0.15)$ & $-31.85(0.32)$ \\
1 & 2 & $-13.60(0.57)$ & $-13.56(0.96)$ \\
1 & 3 & $-40.51(0.41)$ & $-36.13(0.81)$ \\
1 & 4 & $-88.7(2.8)$ & $-83.7(7.9)$ \\
2 & 1 & $-82.6(7.6)$ & $-81(21)$ \\
2 & 2 & $-30.41(0.57)$ & $-28.9(1.4)$ \\
2 & 3 & $-33.89(0.62)$ & $-35.2(3.7)$ \\
4 & 1 & $-73.34(0.48)$ & $-70.2(1.1)$ \\
4 & 2 & $-64.08(0.61)$ & $-67.8(2.2)$ \\
IR6 & 1 & $-39.4(1.6)$ & $-39.4(4.2)$ \\
IR93 & 2 & $-26.07(0.43)$ & $-26.85(0.97)$ \\
IR93 & 3 & $-30.6(1.5)$ & $-32.0(2.5)$ \\
IR93 & 4 & $-29.14(0.77)$ & $-30.3(2.1)$ \\
IR93 & 6 & $-47.7(7.9)$ & $-71(37)$ \\
\hline
\end{tabular}

${ }^{a}$ Measured from $\mathrm{H}_{2}$ 1-0 S(1) $2.1218 \mu m$ line

${ }^{\mathrm{b}}$ Measured from all detected $\mathrm{H}_{2}$ lines fit with model described in section 3.4 
Table 4. Measured properties of $\mathrm{H}_{2}$ flows

\begin{tabular}{|c|c|c|c|c|c|c|c|c|}
\hline Outflow & ${ }^{\text {a }}$ Center & b $\mathrm{PA}$ & c Length & d Source & $\begin{array}{l}\text { e Flow } \\
\text { Length }\end{array}$ & $\begin{array}{l}\text { e Counterflow } \\
\text { Length }\end{array}$ & $\begin{array}{c}\text { f Age } \\
\left(50 \mathrm{~km} \mathrm{~s}^{-1}\right)\end{array}$ & $\begin{array}{l}\text { g LOS } \\
\text { Velocity }\end{array}$ \\
\hline 1 & $05: 39: 13.023+35: 45: 38.66$ & -13.3 & $142.3 "$ & $\mathrm{~mm} 2 ?$ & 58 & 84.2 & $1.4 \mathrm{e} 4$ & - \\
\hline 2 & $05: 39: 13.058+35: 45: 51.28$ & -47.0 & $44.6 ”$ & mm1a & 44.6 & - & $6.6 \mathrm{e} 3$ & Blue \\
\hline 3 & $05: 39: 12.48+35: 45: 54.9$ & -62 & $44 "$ & $\mathrm{~mm} 3 ?$ & 44 & - & $6.5 \mathrm{e} 3$ & Red \\
\hline 4 & ambiguous & $17.8-21.8$ & 141-144" & $?$ & $141-144$ & - & $2.1 \mathrm{e} 4$ & Blue \\
\hline 5 & $05: 39: 12+35: 45: 51$ & 170 & $38-48$ & $\mathrm{~mm} 3 ?$ & $38-48$ & - & $6.5 \mathrm{e} 3$ & Blue \\
\hline 6 & $05: 39: 09.7+35: 45: 17$ & 14.5 & 197 & Q5 & 197 & - & $2.9 \mathrm{e} 4$ & Blue \\
\hline 8 & $05: 39: 10.002+35: 45: 10.87$ & -154.6 & 105.5" & IR41? & 54.7 & 52.9 & $7.9 \mathrm{e} 3$ & - \\
\hline
\end{tabular}

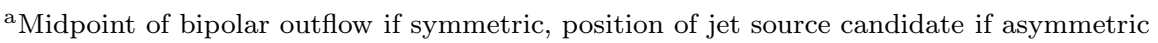

${ }^{\mathrm{b}}$ Position angle uncertainties are $\sim 5^{\circ}$ because they are not perfectly collimated, causing an ambiguity in their true directions. The exact angles used to draw vectors in figure 2 are listed for reproducibility.

cTotal length of outflow on the sky, including counterflow

${ }^{\mathrm{d}}$ Candidate jet source object. Outflows 2 and 6 have clear associations, the others are weaker candidates.

e Flow length is the distance from the CENTER position to the last $\mathrm{H}_{2}$ knot in the position angle direction as listed. Counterflow length is the distance from the CENTER position to the opposite far knot.

${ }^{\mathrm{f}}$ Timescale of jet assuming it is propagating at $50 \mathrm{~km} \mathrm{~s}^{-1}$, an effective lower limit to see $\mathrm{H}_{2}$ emission. If two lengths are available, uses the longer of the two. These are lower limits to the true timescale (Parker et al. 1991).

g The parity of the outflow along the line of sight. Outflow 1 and 8 have counterflows with parities as indicated in figure 2 
Table 5. Measured $\mathrm{H}_{2}$ line strengths

\begin{tabular}{|c|c|c|c|c|c|c|c|c|c|c|c|c|}
\hline aperture & $\begin{array}{c}1-0 \mathrm{~S}(0) \\
2.2233\end{array}$ & $\begin{array}{l}1-0 \mathrm{~S}(1) \\
2.12183\end{array}$ & $\begin{array}{l}1-0 \mathrm{~S}(2) \\
2.03376\end{array}$ & $\begin{array}{l}1-0 \mathrm{~S}(3) \\
1.95756\end{array}$ & $\begin{array}{l}1-0 \mathrm{~S}(6) \\
1.78795\end{array}$ & $\begin{array}{l}1-0 \mathrm{~S}(7) \\
1.74803\end{array}$ & $\begin{array}{l}1-0 \mathrm{~S}(8) \\
1.71466\end{array}$ & $\begin{array}{l}1-0 \mathrm{~S}(9) \\
1.68772\end{array}$ & $\begin{array}{c}1-0 \mathrm{Q}(1) \\
2.40659\end{array}$ & $\begin{array}{c}1-0 \mathrm{Q}(2) \\
2.41344\end{array}$ & $\begin{array}{c}1-0 \mathrm{Q}(3) \\
2.42373\end{array}$ & $\begin{array}{c}1-0 \mathrm{Q}(4) \\
2.43749\end{array}$ \\
\hline outflow1ap1 & $\begin{array}{c}3.60 \mathrm{E}-15 \\
(2.4 \mathrm{e}-17)\end{array}$ & $\begin{array}{l}9.80 \mathrm{E}-15 \\
(3.4 \mathrm{e}-17)\end{array}$ & $\begin{array}{l}5.50 \mathrm{E}-15 \\
(6.8 \mathrm{e}-17)\end{array}$ & $\begin{array}{c}1.20 \mathrm{E}-14 \\
(2 \mathrm{e}-16)\end{array}$ & $\begin{array}{c}4.70 \mathrm{E}-15 \\
(2 \mathrm{e}-16)\end{array}$ & $\begin{array}{c}3.10 \mathrm{E}-15 \\
(2.8 \mathrm{e}-17)\end{array}$ & $\begin{array}{l}8.60 \mathrm{E}-16 \\
(2.8 \mathrm{e}-17)\end{array}$ & $\begin{array}{c}1.10 \mathrm{E}-15 \\
(2.7 \mathrm{e}-17)\end{array}$ & $\begin{array}{l}9.20 \mathrm{E}-15 \\
(1.4 \mathrm{e}-16)\end{array}$ & $\begin{array}{c}6.10 \mathrm{E}-15 \\
(7.4 \mathrm{e}-17)\end{array}$ & $\begin{array}{l}1.10 \mathrm{E}-14 \\
(8.8 \mathrm{e}-17)\end{array}$ & $\begin{array}{c}6.90 \mathrm{E}-15 \\
(7.8 \mathrm{e}-17)\end{array}$ \\
\hline outflow1ap2 & $\begin{array}{c}7.10 \mathrm{E}-16 \\
(2.1 \mathrm{e}-17)\end{array}$ & $\begin{array}{c}1.80 \mathrm{E}-15 \\
(2.7 \mathrm{e}-17)\end{array}$ & $\begin{array}{l}9.90 \mathrm{E}-16 \\
(6.8 \mathrm{e}-17)\end{array}$ & $\begin{array}{c}1.80 \mathrm{E}-15 \\
(1.7 \mathrm{e}-16)\end{array}$ & - & $\begin{array}{l}- \\
-\end{array}$ & $\begin{array}{l}- \\
-\end{array}$ & $\begin{array}{l}- \\
-\end{array}$ & $\begin{array}{c}3.00 \mathrm{E}-15 \\
(1.3 \mathrm{e}-16)\end{array}$ & $\begin{array}{c}1.90 \mathrm{E}-15 \\
(4 \mathrm{e}-17)\end{array}$ & $\begin{array}{l}3.20 \mathrm{E}-15 \\
(7.8 \mathrm{e}-17)\end{array}$ & $\begin{array}{r}2.00 \mathrm{E}-15 \\
(3.8 \mathrm{e}-17)\end{array}$ \\
\hline outflow1ap3 & $\begin{array}{l}1.60 \mathrm{E}-15 \\
(2.4 \mathrm{e}-17)\end{array}$ & $\begin{array}{l}4.10 \mathrm{E}-15 \\
(3.4 \mathrm{e}-17)\end{array}$ & $\begin{array}{l}2.20 \mathrm{E}-15 \\
(6.3 \mathrm{e}-17)\end{array}$ & $\begin{array}{r}4.70 \mathrm{E}-15 \\
(1.8 \mathrm{e}-16)\end{array}$ & - & $\begin{array}{l}8.30 \mathrm{E}-16 \\
(2.8 \mathrm{e}-17)\end{array}$ & - & - & $\begin{array}{l}5.60 \mathrm{E}-15 \\
(1.4 \mathrm{e}-16)\end{array}$ & $\begin{array}{c}3.70 \mathrm{E}-15 \\
(5.9 \mathrm{e}-17)\end{array}$ & $\begin{array}{l}6.60 \mathrm{E}-15 \\
(8.2 \mathrm{e}-17)\end{array}$ & $\begin{array}{r}4.80 \mathrm{E}-15 \\
(5.2 \mathrm{e}-17)\end{array}$ \\
\hline outflow1ap4 & $\begin{array}{l}- \\
-\end{array}$ & $\begin{array}{c}9.00 \mathrm{E}-16 \\
(3 \mathrm{e}-17)\end{array}$ & $\begin{array}{l}- \\
-\end{array}$ & $\begin{array}{l}- \\
-\end{array}$ & - & $\begin{array}{l}- \\
-\end{array}$ & - & - & $\begin{array}{l}- \\
-\end{array}$ & $\begin{array}{l}- \\
-\end{array}$ & $\begin{array}{l}- \\
-\end{array}$ & $\begin{array}{l}- \\
-\end{array}$ \\
\hline outflow2ap1 & - & $\begin{array}{c}3.60 \mathrm{E}-16 \\
(1.5 \mathrm{e}-17)\end{array}$ & $\begin{array}{l}- \\
-\end{array}$ & $\begin{array}{l}- \\
-\end{array}$ & - & $\begin{array}{l}- \\
-\end{array}$ & - & - & $\begin{array}{l}- \\
-\end{array}$ & - & $\begin{array}{l}- \\
-\end{array}$ & - \\
\hline outflow2ap2 & $\begin{array}{c}9.40 \mathrm{E}-16 \\
(1.7 \mathrm{e}-17)\end{array}$ & $\begin{array}{l}2.40 \mathrm{E}-15 \\
(2.2 \mathrm{e}-17)\end{array}$ & $\begin{array}{c}1.50 \mathrm{E}-15 \\
(5.8 \mathrm{e}-17)\end{array}$ & $\begin{array}{c}1.80 \mathrm{E}-15 \\
(1.1 \mathrm{e}-16)\end{array}$ & - & $\begin{array}{l}4.00 \mathrm{E}-16 \\
(2.3 \mathrm{e}-17)\end{array}$ & - & - & $\begin{array}{c}3.00 \mathrm{E}-15 \\
(4.7 \mathrm{e}-17)\end{array}$ & - & $\begin{array}{l}3.70 \mathrm{E}-15 \\
(7.9 \mathrm{e}-17)\end{array}$ & - \\
\hline outflow2ap3 & $\begin{array}{l}2.10 \mathrm{E}-15 \\
(1.7 \mathrm{e}-17)\end{array}$ & $\begin{array}{l}1.90 \mathrm{E}-15 \\
(2.2 \mathrm{e}-17)\end{array}$ & $\begin{array}{c}1.80 \mathrm{E}-15 \\
(5.1 \mathrm{e}-17)\end{array}$ & $\begin{array}{c}2.20 \mathrm{E}-15 \\
(1.3 \mathrm{e}-16)\end{array}$ & - & $\begin{array}{l}6.70 \mathrm{E}-16 \\
(2.9 \mathrm{e}-17)\end{array}$ & $\begin{array}{l}- \\
-\end{array}$ & $\begin{array}{l}- \\
-\end{array}$ & $\begin{array}{c}5.70 \mathrm{E}-15 \\
-8.00 \mathrm{E}-16\end{array}$ & - & $\begin{array}{c}7.30 \mathrm{E}-15 \\
-8.00 \mathrm{E}-16\end{array}$ & $\begin{array}{l}- \\
-\end{array}$ \\
\hline outflow4ap1 & $\begin{array}{c}5.50 \mathrm{E}-16 \\
(2 \mathrm{e}-17)\end{array}$ & $\begin{array}{c}2.00 \mathrm{E}-15 \\
(2 \mathrm{e}-17)\end{array}$ & $\begin{array}{c}8.50 \mathrm{E}-16 \\
(5 \mathrm{e}-17)\end{array}$ & $\begin{array}{r}2.00 \mathrm{E}-15 \\
(1.3 \mathrm{e}-16)\end{array}$ & - & $\begin{array}{l}9.40 \mathrm{E}-16 \\
(2.8 \mathrm{e}-17)\end{array}$ & $\begin{array}{l}1.90 \mathrm{E}-16 \\
(1.8 \mathrm{e}-17)\end{array}$ & $\begin{array}{l}3.40 \mathrm{E}-16 \\
(2.3 \mathrm{e}-17)\end{array}$ & $\begin{array}{c}1.40 \mathrm{E}-15 \\
-4.00 \mathrm{E}-16\end{array}$ & - & $\begin{array}{l}1.40 \mathrm{E}-15 \\
(6.9 \mathrm{e}-17)\end{array}$ & $\begin{array}{l}- \\
-\end{array}$ \\
\hline outflow 4 ap2 & $\begin{array}{c}5.60 \mathrm{E}-16 \\
(2 \mathrm{e}-17)\end{array}$ & $\begin{array}{c}2.00 \mathrm{E}-15 \\
(2.2 \mathrm{e}-17)\end{array}$ & $\begin{array}{l}5.30 \mathrm{E}-16 \\
(2.4 \mathrm{e}-17)\end{array}$ & $\begin{array}{c}2.10 \mathrm{E}-15 \\
(1.2 \mathrm{e}-16)\end{array}$ & - & $\begin{array}{l}5.80 \mathrm{E}-16 \\
(2.3 \mathrm{e}-17)\end{array}$ & $\begin{array}{l}- \\
-\end{array}$ & $\begin{array}{l}1.10 \mathrm{E}-16 \\
(1.8 \mathrm{e}-17)\end{array}$ & $\begin{array}{l}- \\
-\end{array}$ & - & $\begin{array}{c}2.00 \mathrm{E}-15 \\
-2.00 \mathrm{E}-16\end{array}$ & $\begin{array}{l}- \\
-\end{array}$ \\
\hline IR6ap1 & $\begin{array}{l}- \\
-\end{array}$ & $\begin{array}{c}1.10 \mathrm{E}-15 \\
(3 \mathrm{e}-17)\end{array}$ & $\begin{array}{l}- \\
-\end{array}$ & $\begin{array}{c}9.30 \mathrm{E}-16 \\
(1.4 \mathrm{e}-16)\end{array}$ & $\begin{array}{l}- \\
-\end{array}$ & $\begin{array}{l}4.30 \mathrm{E}-16 \\
(3.2 \mathrm{e}-17)\end{array}$ & - & $\begin{array}{l}- \\
-\end{array}$ & - & - & $\begin{array}{l}- \\
-\end{array}$ & - \\
\hline IR93ap1 & $\begin{array}{l}- \\
-\end{array}$ & $\begin{array}{l}6.60 \mathrm{E}-15 \\
(3.5 \mathrm{e}-17)\end{array}$ & $\begin{array}{l}- \\
-\end{array}$ & $\begin{array}{c}2.70 \mathrm{E}-15 \\
(1 \mathrm{e}-16)\end{array}$ & $\begin{array}{l}- \\
-\end{array}$ & $\begin{array}{l}- \\
-\end{array}$ & $\begin{array}{l}- \\
-\end{array}$ & - & $\begin{array}{l}- \\
-\end{array}$ & $\begin{array}{l}- \\
-\end{array}$ & $\begin{array}{l}5.80 \mathrm{E}-15 \\
(7.4 \mathrm{e}-17)\end{array}$ & $\begin{array}{l}- \\
-\end{array}$ \\
\hline IR93ap2 & $\begin{array}{l}4.40 \mathrm{E}-15 \\
(3.2 \mathrm{e}-17)\end{array}$ & $\begin{array}{l}6.60 \mathrm{E}-15 \\
(3.7 \mathrm{e}-17)\end{array}$ & $\begin{array}{c}3.90 \mathrm{E}-15 \\
(9.2 \mathrm{e}-17)\end{array}$ & $\begin{array}{c}3.30 \mathrm{E}-15 \\
(1.4 \mathrm{e}-16)\end{array}$ & $\begin{array}{l}- \\
-\end{array}$ & $\begin{array}{l}1.10 \mathrm{E}-15 \\
(2.7 \mathrm{e}-17)\end{array}$ & $\begin{array}{l}- \\
-\end{array}$ & $\begin{array}{l}- \\
-\end{array}$ & $\begin{array}{c}7.60 \mathrm{E}-15 \\
(8 \mathrm{e}-17)\end{array}$ & $\begin{array}{c}5.10 \mathrm{E}-15 \\
(5.2 \mathrm{e}-17)\end{array}$ & $\begin{array}{l}6.90 \mathrm{E}-15 \\
(7.4 \mathrm{e}-17)\end{array}$ & $\begin{array}{c}5.50 \mathrm{E}-15 \\
(6.1 \mathrm{e}-17)\end{array}$ \\
\hline IR93ap3 & $\begin{array}{c}1.00 \mathrm{E}-15 \\
(2.3 \mathrm{e}-17)\end{array}$ & $\begin{array}{l}1.70 \mathrm{E}-15 \\
(3.6 \mathrm{e}-17)\end{array}$ & $\begin{array}{l}- \\
-\end{array}$ & $\begin{array}{l}9.00 \mathrm{E}-16 \\
(1.2 \mathrm{e}-16)\end{array}$ & - & $\begin{array}{l}- \\
-\end{array}$ & $\begin{array}{l}- \\
-\end{array}$ & $\begin{array}{l}- \\
-\end{array}$ & $\begin{array}{c}2.00 \mathrm{E}-15 \\
(8 \mathrm{e}-17)\end{array}$ & $\begin{array}{c}1.70 \mathrm{E}-15 \\
(3.8 \mathrm{e}-17)\end{array}$ & $\begin{array}{l}1.90 \mathrm{E}-15 \\
(8.8 \mathrm{e}-17)\end{array}$ & $\begin{array}{l}- \\
-\end{array}$ \\
\hline IR93ap4 & $\begin{array}{l}2.60 \mathrm{E}-15 \\
(3.2 \mathrm{e}-17)\end{array}$ & $\begin{array}{l}3.70 \mathrm{E}-15 \\
(3.6 \mathrm{e}-17)\end{array}$ & $\begin{array}{l}- \\
-\end{array}$ & $\begin{array}{l}- \\
-\end{array}$ & - & - & $\begin{array}{l}- \\
-\end{array}$ & $\begin{array}{l}- \\
-\end{array}$ & $\begin{array}{l}4.30 \mathrm{E}-15 \\
(8.5 \mathrm{e}-17)\end{array}$ & $\begin{array}{c}3.50 \mathrm{E}-15 \\
(5.2 \mathrm{e}-17)\end{array}$ & $\begin{array}{l}4.70 \mathrm{E}-15 \\
(7.4 \mathrm{e}-17)\end{array}$ & - \\
\hline IR93ap5 & $\begin{array}{l}- \\
-\end{array}$ & $\begin{array}{l}1.90 \mathrm{E}-15 \\
(2.4 \mathrm{e}-17)\end{array}$ & - & $\begin{array}{c}1.00 \mathrm{E}-15 \\
(1.00 \mathrm{e}-16)\end{array}$ & $\begin{array}{l}- \\
-\end{array}$ & - & - & $\begin{array}{l}- \\
-\end{array}$ & $\begin{array}{l}- \\
-\end{array}$ & - & $\begin{array}{l}- \\
-\end{array}$ & - \\
\hline IR93ap6 & $\begin{array}{l}- \\
-\end{array}$ & $\begin{array}{c}4.10 \mathrm{E}-16 \\
(3 \mathrm{e}-17)\end{array}$ & $\begin{array}{l}- \\
-\end{array}$ & $\begin{array}{l}- \\
-\end{array}$ & $\begin{array}{l}- \\
-\end{array}$ & - & - & $\begin{array}{l}- \\
-\end{array}$ & $\begin{array}{l}- \\
-\end{array}$ & - & - & $\begin{array}{l}- \\
-\end{array}$ \\
\hline
\end{tabular}

Note. - Fluxes are in units erg s s $\mathrm{cm}^{-2} \AA^{-1}$. Errors are listed on the second row for each aperture. Errors of $(0)$ indicate that the line was detected, but that the fluxes should not be trusted because the background was probably oversubtracted. 
Table 5. Measured $\mathrm{H}_{2}$ line strengths (cont'd)

\begin{tabular}{|c|c|c|c|c|c|c|c|}
\hline & $\begin{array}{l}2-1 \mathrm{~S}(1) \\
2.24772\end{array}$ & $\begin{array}{l}2-1 \mathrm{~S}(3) \\
2.07351\end{array}$ & $\begin{array}{c}3-2 \mathrm{~S}(3) \\
2.2014\end{array}$ & $\begin{array}{l}3-2 \mathrm{~S}(4) \\
2.12797\end{array}$ & $\begin{array}{l}4-3 \mathrm{~S}(5) \\
2.20095\end{array}$ & $\begin{array}{l}{[\mathrm{Fe} \mathrm{II}]} \\
1.6435\end{array}$ & $\begin{array}{l}{[\mathrm{Fe} \text { II }]} \\
1.2567\end{array}$ \\
\hline outflow1ap1 & $\begin{array}{c}2.00 \mathrm{E}-15 \\
(2.5 \mathrm{e}-17)\end{array}$ & $\begin{array}{c}1.20 \mathrm{E}-15 \\
(3.5 \mathrm{e}-17)\end{array}$ & $\begin{array}{c}6.20 \mathrm{E}-16 \\
(2.2 \mathrm{e}-17)\end{array}$ & $\begin{array}{c}2.60 \mathrm{E}-16 \\
(1.6 \mathrm{e}-17)\end{array}$ & $\begin{array}{c}7.10 \mathrm{E}-16 \\
(1.9 \mathrm{e}-17)\end{array}$ & $\begin{array}{c}4.4 \mathrm{e}-15 \\
(7.9 \mathrm{e}-17)\end{array}$ & $\begin{array}{l}3.5 \mathrm{e}-15 \\
(4 \mathrm{e}-17)\end{array}$ \\
\hline outflow1ap2 & $\begin{array}{l}- \\
-\end{array}$ & $\begin{array}{l}- \\
-\end{array}$ & $\begin{array}{l}- \\
-\end{array}$ & $\begin{array}{l}- \\
-\end{array}$ & $\begin{array}{l}- \\
-\end{array}$ & $\begin{array}{c}6.7 \mathrm{e}-16 \\
(7.8 \mathrm{e}-17)\end{array}$ & $\begin{array}{c}3.1 \mathrm{e}-16 \\
(3.3 \mathrm{e}-17)\end{array}$ \\
\hline outflow1ap3 & $\begin{array}{c}9.90 \mathrm{E}-16 \\
(2.6 \mathrm{e}-17)\end{array}$ & $\begin{array}{l}- \\
-\end{array}$ & $\begin{array}{c}5.70 \mathrm{E}-16 \\
(0)\end{array}$ & $\begin{array}{c}2.50 \mathrm{E}-16 \\
(1.2 \mathrm{e}-17)\end{array}$ & $\begin{array}{c}6.40 \mathrm{E}-16 \\
(0)\end{array}$ & $\begin{array}{c}1.3 \mathrm{e}-15 \\
(8.9 \mathrm{e}-17)\end{array}$ & $\begin{array}{l}5.7 \mathrm{e}-16 \\
(4 \mathrm{e}-17)\end{array}$ \\
\hline outflow1ap4 & $\begin{array}{l}- \\
-\end{array}$ & - & - & $\begin{array}{l}- \\
-\end{array}$ & - & $\begin{array}{c}(0.5 e-11) \\
- \\
-\end{array}$ & $\begin{array}{c}(4 \mathrm{e}-11) \\
- \\
-\end{array}$ \\
\hline outflow2ap1 & - & - & - & - & - & - & - \\
\hline outflow2ap2 & $\begin{array}{c}6.40 \mathrm{E}-16 \\
(1.9 \mathrm{e}-17)\end{array}$ & - & - & - & - & - & - \\
\hline outflow2ap3 & $\begin{array}{l}- \\
-\end{array}$ & - & - & - & - & - & - \\
\hline outflow4ap1 & $\begin{array}{c}4.30 \mathrm{E}-16 \\
(1.9 \mathrm{e}-17)\end{array}$ & $\begin{array}{c}4.20 \mathrm{E}-16 \\
(0)\end{array}$ & - & - & $\begin{array}{c}1.60 \mathrm{E}-16 \\
(0)\end{array}$ & - & - \\
\hline outflow4ap2 & $\begin{array}{l}- \\
-\end{array}$ & $\begin{array}{l}- \\
-\end{array}$ & - & - & - & - & - \\
\hline IR6ap1 & - & - & - & - & - & - & - \\
\hline IR93ap1 & - & - & - & - & - & - & - \\
\hline IR93ap2 & $\begin{array}{c}3.80 \mathrm{E}-15 \\
(2.2 \mathrm{e}-17)\end{array}$ & $\begin{array}{l}- \\
- \\
-\end{array}$ & $\begin{array}{c}3.10 \mathrm{E}-15 \\
(0)\end{array}$ & $\begin{array}{l}- \\
-\end{array}$ & $\begin{array}{l}- \\
-\end{array}$ & $\begin{array}{l}- \\
- \\
-\end{array}$ & $\begin{array}{l}- \\
-\end{array}$ \\
\hline IR93ap3 & - & - & - & - & - & - & - \\
\hline & - & - & - & - & - & - & - \\
\hline IR93ap4 & - & - & - & - & - & - & - \\
\hline IR93ap5 & - & - & - & - & - & - & - \\
\hline & - & - & - & - & - & - & - \\
\hline IR93ap6 & - & - & - & - & - & - & - \\
\hline & - & - & - & - & - & - & - \\
\hline
\end{tabular}

Note. - Fluxes are in units erg s $\mathrm{s}^{-1} \mathrm{~cm}^{-2} \AA^{-1}$. Errors are listed on the second row for each aperture. Errors of (0) indicate that the line was detected, but that the fluxes should not be trusted because the background was probably oversubtracted. 


\subsection{Spectroscopic Results: Optical}

IR 6 and IR 41 (objects 1 and 6 in Figure 2) both show $\mathrm{H} \alpha$ in emission. IR 41 is close to the reflection nebula in the southeast portion of IRAS 05358 and is probably the reflected star. The reflection nebula's spectrum is very similar to IR 41's spectrum at $\mathrm{H} \alpha$ in both width and brightness (see Figure 12).

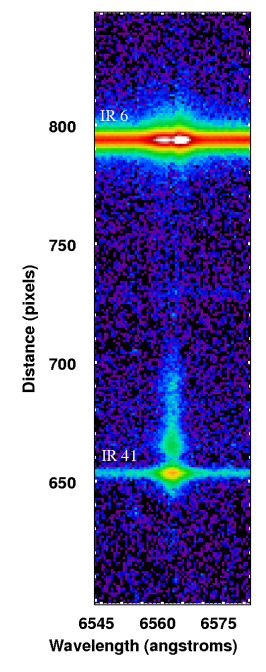

Fig. 12.- A position-velocity diagram of IR 6 and 41 including the reflection nebula near IR 41 $\left(\approx 7.4 M_{\odot}\right)$. IR 6 shows a two-peaked $\mathrm{H} \alpha$ emission profile, but is the less massive $\left(\approx 4.5 M_{\odot}\right)$ of the pair. The separation between the two sources is $55^{\prime \prime} 3$, and each pixel is 0.4 .

There are three components in the $\mathrm{H} \alpha$ profile of IR 6: a broad absorption feature seen far $(\sim$ $400 \mathrm{~km} \mathrm{~s}^{-1}$ from the line center) on the wings and two emission peaks. The peaks are separated by $190 \mathrm{~km} \mathrm{~s}^{-1}$ and the blueshifted peak is weaker than the redshifted (Table 6). The $\mathrm{H} \beta$ profile shows much deeper absorption and weaker emission but with similar characteristics. The presence of the $\mathrm{H} \alpha$ emission makes identification of the stellar type from the $\mathrm{H} \alpha$ line profile uncertain. The derived extinction to IR 6 is at least $A_{V}=7$ from an assumed $\mathrm{H} \alpha / \mathrm{H} \beta$ ratio of 2.87 (Osterbrock \& Ferland 2006). The $\mathrm{H} \beta$ flux was measured from zero to the peaks of the emission profile and therefore probably overestimates the $\mathrm{H} \beta$ flux and underestimates the extinction.

\subsection{Radio Interferometry}

A point source was detected in the X, U, K and Q band VLA maps with high significance at the same location as the X-band point source reported in Beuther et al. (2007). Seven-parameter gaussians were fit to each image to measure the beam sizes and positions and flux densities. The 
Table 6. IR 6 Deblended Profiles

\begin{tabular}{cccccccc}
\hline \hline & $\begin{array}{c}\mathrm{a} \text { Blue } \\
\text { Emission }\end{array}$ & $\begin{array}{c}\mathrm{b} \text { Blue } \\
\text { Wavelength }\end{array}$ & $\begin{array}{c}{ }^{\mathrm{a}} \text { Red } \\
\text { Emission }\end{array}$ & $\begin{array}{c}\mathrm{b} \text { Red } \\
\text { Wavelength }\end{array}$ & Absorption & $\begin{array}{c}\text { Gaussian / } \\
\text { Lorentzian FWHM }\end{array}$ & $\begin{array}{c}\mathrm{b} \text { Absorption } \\
\text { Wavelength }\end{array}$ \\
\hline $\mathrm{H} \alpha$ & $\begin{array}{c}4.4 \times 10^{-14} \\
\mathrm{H} \beta\end{array}$ & $\begin{array}{c}\mathrm{c} 2.4 \times 10^{-14} \\
4859.79\end{array}$ & $\begin{array}{c}1.3 \times 10^{-13} \\
\mathrm{c} 1.8 \times 10^{-14}\end{array}$ & $\begin{array}{c}6564.23 \\
4864.28\end{array}$ & $\begin{array}{c}-2.6 \times 10^{-14} \\
\mathrm{~d}-4.6 \times 10^{-14}\end{array}$ & $\begin{array}{c}1.5 / 0.19 \\
0.17 / 16.5\end{array}$ & 6563.02 \\
\hline
\end{tabular}

Note. - Measurements are made using a Voigt profile fit in the IRAF SPLOT task.

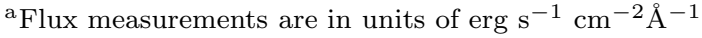

${ }^{\mathrm{b}}$ Wavelengths are in Geocentric coordinates. Subtract $0.53 \AA$ from $\mathrm{H} \alpha$ and $0.39 \AA$ from $\mathrm{H} \beta$ to put in LSR coordinates.

${ }^{\mathrm{c}} \mathrm{H} \beta$ emission was measured assuming a continuum of zero and therefore represents an upper limit in the $\mathrm{H} \beta$ emission

${ }^{\mathrm{d}} \mathrm{H} \beta$ deblending may contain systematic errors from a guessed subtraction of the $\mathrm{H} \beta$ emission

Table 7. Lines observed in the optical spectra

\begin{tabular}{|c|c|c|c|c|c|c|c|}
\hline Source & $\mathrm{H} \alpha$ & $\mathrm{H} \beta$ & [S II] $6716 \AA$ & [S II] $6731 \AA$ & [O I] $6300 \AA$ & [O I] $6363 \AA$ & [O I] $5577 \AA$ \\
\hline \multirow[t]{2}{*}{ Outflow1 ap1 } & $4.3 \times 10^{-16}$ & - & $5.7 \times 10^{-16}$ & $6.3 \times 10^{-16}$ & $5.3 \times 10^{-16}$ & $1.8 \times 10^{-16}$ & - \\
\hline & 6561.49 & - & 6715.3 & 6729.6 & 6299.7 & 6363.3 & - \\
\hline \multirow[t]{2}{*}{ Outflow1 ap2 } & $4.5 \times 10^{-16}$ & - & $4.5 \times 10^{-16}$ & $4.6 \times 10^{-16}$ & $3.1 \times 10^{-16}$ & $1.2 \times 10^{-16}$ & - \\
\hline & 6561.22 & - & 6714.9 & 6729.3 & 6299.4 & 6363.2 & - \\
\hline \multirow[t]{2}{*}{ Ambient Medium - slit 1} & $6.7 \times 10^{-17}$ & $5.3 \times 10^{-18}$ & $1.0 \times 10^{-17}$ & $7.9 \times 10^{-18}$ & $3.5 \times 10^{-16}$ & $1.2 \times 10^{-16}$ & $4.8 \times 10^{-17}$ \\
\hline & 6562.87 & 4861.7 & 6716.7 & 6731.2 & 6300.3 & 6363.8 & 5578.0 \\
\hline \multirow[t]{2}{*}{ IR 41 nebula } & $2.6 \times 10^{-15}$ & - & - & - & $4.4 \times 10^{-16}$ & $1.9 \times 10^{-16}$ & - \\
\hline & 6562.85 & - & - & - & 6300.3 & 6363.9 & - \\
\hline \multirow[t]{2}{*}{ IR 41} & $6.5 \times 10^{-15}$ & - & - & - & $1.1 \times 10^{-16}$ & $7 \times 10^{-17}$ & - \\
\hline & 6562.9 & - & - & - & 6300.0 & 6363.3 & - \\
\hline \multirow[t]{2}{*}{ IR 6} & $1.76 \times 10^{-13}$ & a $4.1 \times 10^{-14}$ & - & - & - & - & \\
\hline & - & - & - & - & - & - & \\
\hline
\end{tabular}

Note. - Wavelengths listed are in $\AA$ and are geocentric. To convert to LSR velocities, subtract $24.35 \mathrm{~km} \mathrm{~s}^{-1}$. The ambient medium fluxes represent averages across the slit. Fluxes are in $\operatorname{erg} \mathrm{s}^{-1} \mathrm{~cm}^{-2} \AA^{-1}$.

${ }^{\mathrm{a}} \mathrm{H} \beta$ measurement in IR 6 is an upper limit 
measurements are listed in Table 8 . The locations of the point source and the shape of the beams from the re-reduced X and Q band images are displayed in figure 13 . A Class II $6.7 \mathrm{GHz}$ methanol maser was detected in IRAS 05358 by Menten (1991). It was observed with the European VLBI Network (EVN) by Minier et al. (2000) and seen to consist of a linear string of maser spots that trace a probable disk in addition to maser spots scattered around a line perpendicular to the proposed disk (see Figure 13). The VLA source is more than a VLA beam away from the VLBI $\mathrm{CH}_{3} \mathrm{OH}$ maser disk identified by Minier et al. (2000). It is to the southeast in the opposite direction of Outflow 2. Outflow 2 is at position angle $-47^{\circ}$, while the disk is at PA $25^{\circ}$. The $8^{\circ}$ difference from being perpendicular is well within the error associated with determining the angle of the outflow in this confused region, so the VLBI disk is a strong candidate for the source of Outflow 2.

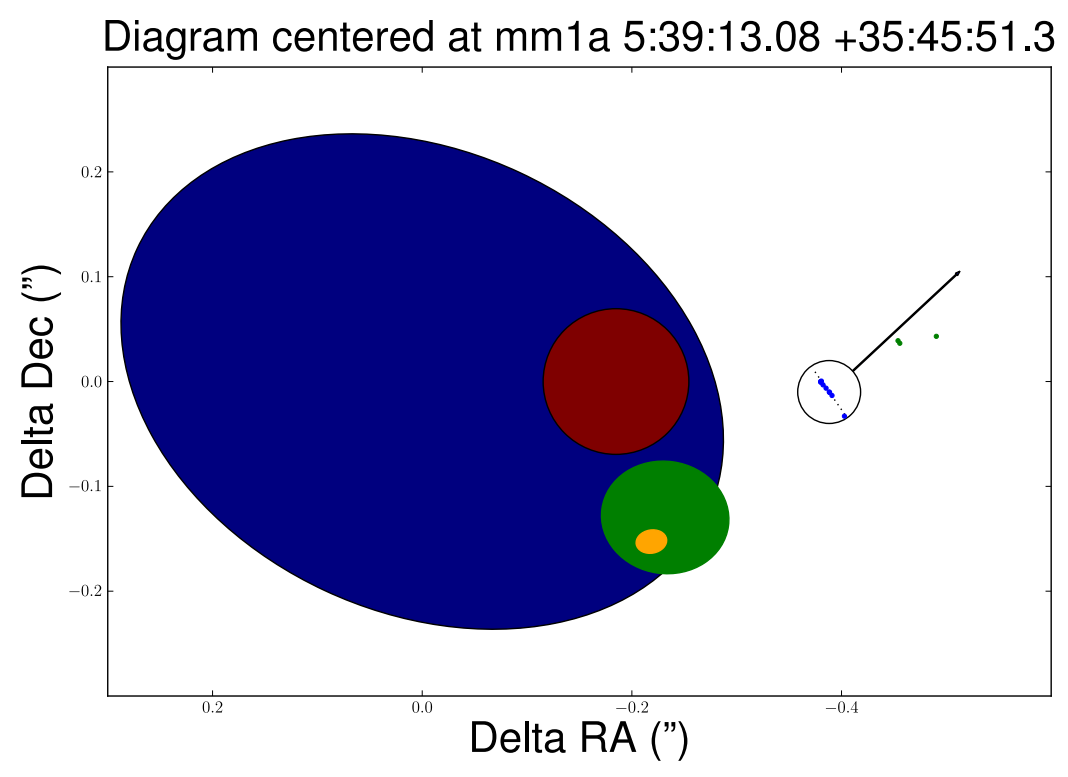

Fig. 13.- A diagram of the region surrounding mm1a from Beuther et al. (2007). The ellipses are centered at the measured source centers and their sizes represent the beam sizes of the Plateau de Bure interferometer at $1.2 \mathrm{~mm}$ (blue, Beuther et al. 2007), Gemini MICHELLE at 7.9 $\mu \mathrm{m}$ (red, Longmore et al. 2006), the VLA at $3.6 \mathrm{~cm}$ (green), and the VLA at $7 \mathrm{~mm}$ (orange). The maser disk was measured with the European VLBI Network by Minier et al. (2000), so the size and direction of the disk are very well constrained. The black circle is centered on the pointing center of the VLBI observation and represents the absolute pointing uncertainty. The arrow pointing in the direction of Outflow 2 traces clumps along the outflow back to the mm emission region. The vector is not to scale - Outflow 2 is about $45^{\prime \prime}$ long.

The astrometric uncertainty in VLA measurements are typically $\lesssim 0.1^{\prime \prime}$. Different epochs of high-resolution X-band and Q-band data confirmed that the pointing accuracy is substantially better than $0.1^{\prime \prime}$ in this case. The VLBI absolute pointing uncertainty is reported to have an upper limit of $\sim 0.03^{\prime \prime}$ (Minier et al. 2000). The separation between the VLA Q-band center and the VLBI 
disk center is $0.22^{\prime \prime}$, whereas the separation between the combined $\mathrm{X}$ and Q band pointing centers is only $0.027^{\prime \prime}$, which can be viewed as a characteristic uncertainty. This is evidence for at least two distinct massive stars in a binary separated by $\sim 400 \mathrm{AU}$. While the statistical significance of the binary separation is quite high using formal errors, the systematic errors cannot be constrained nearly as well. This object is a candidate binary system but is not yet confirmed.

\section{Analysis}

\subsection{Near-Infrared Spectroscopic Extinction Measurements}

Extinction along a line of sight can be calculated using the 1-0 Q(3) / 1-0 S(1) line ratio.

$$
A_{\lambda}=1.09\left[-\ln \frac{S_{\nu}(S) / S_{\nu}(Q)}{A_{u l}(S) \lambda_{Q} / A_{u l}(Q) \lambda_{S}}\right]\left[\left(\frac{\lambda_{S}}{\lambda_{Q}}\right)^{-1.8}-1\right]^{-1}
$$

Because they are from the same upper state, their intensity ratio should be set by their Einstein A values times the relative energies of the transitions. However, as shown by Luhman et al. (1998), narrow atmospheric absorption lines in the long wavelength portion of the $\mathrm{K}$ band, where the Q branch lines lie, can create a significant bias. Because the lines have not been corrected for atmospheric absorption, the Q branch fluxes should actually be lower limits. Since the 1-0 S(1)

Table 8. VLA measurements near IRAS $05358 \mathrm{~mm} 1 \mathrm{a}$

\begin{tabular}{|c|c|c|c|c|}
\hline $\begin{array}{l}\text { Frequency } \\
\text { Observed }\end{array}$ & $\begin{array}{l}\text { Beam major / } \\
\text { minor / PA }\end{array}$ & $\begin{array}{l}\text { RA (error) } \\
\text { Dec (error) }\end{array}$ & $\begin{array}{l}\text { Peak flux } \\
\text { (error) }\end{array}$ & $\begin{array}{c}\text { Map RMS } \\
(\mathrm{mJy} / \text { beam })\end{array}$ \\
\hline $43.3 \mathrm{GHz}$ & $0.022^{\prime \prime} / 0.029^{\prime \prime} /-10.4^{\circ}$ & $\begin{array}{l}05: 39: 13.065425(0.000015) \\
35: 45: 51.14732(0.00031)\end{array}$ & $1.319(0.027)$ & 0.179 \\
\hline $22.5 \mathrm{GHz}$ & $1.52^{\prime \prime} / 1.28^{\prime \prime} / 232^{\circ}$ & $\begin{array}{l}05: 39: 13.05521(0.0029) \\
35: 45: 51.378(0.046)\end{array}$ & $1.26(0.04)$ & 0.091 \\
\hline $15.0 \mathrm{GHz}$ & $1.58^{\prime \prime} / 2.00^{\prime \prime} / 0^{\circ}$ & $\begin{array}{l}05: 39: 13.062(0.005) \\
35: 45: 51.4(0.1)\end{array}$ & $1.274(0.065)$ & 0.124 \\
\hline $8.4 \mathrm{GHz}$ & $0.107^{\prime \prime} / 0.122^{\prime \prime} / 7.9^{\circ}$ & $\begin{array}{ll}05: 39: 13.064548 & (0.000036) \\
35: 45: 51.170356 & (0.000613)\end{array}$ & $0.506(0.003)$ & 0.015 \\
\hline
\end{tabular}

-Errors reported here are fit errors. Absolute flux calibration errors are negligible for the X-band data but are about equivalent to measurement errors for the $\mathrm{K}$, and $\mathrm{U}$ bands and dominant in the Q band 
transition at 2.1818 microns is affected very little by atmospheric absorption, and the exinction measured is proportional to the $\mathrm{Q} / \mathrm{S}$ line ratio, the measured extinction should be a lower limit.

The [Fe II] 1.6435 and $1.2567 \mu \mathrm{m}$ lines were detected in Outflow 1, allowing for another direct measurement of the extinction. The measured ratio $F R=1.26 \mu \mathrm{m} / 1.64 \mu \mathrm{m}$ in Outflow 1 was .8, while the true value is at least 1.24 but may be as high as 1.49 (Smith \& Hartigan 2006; Luhman et al. 1998; Giannini et al. 2008). The extinction measured from this ratio ranges from $A_{V}=4.1(F R=1.24)$ to $5.8(F R=1.49)$. The $\mathrm{S}(1) / \mathrm{Q}(3)$ ratio uncorrected for telluric absorption is .91, which yields an extinction lower limit of $A_{V}=18.7$, is inconsistent with the measurement from [Fe II]. The $\mathrm{H} \alpha$ detection and $\mathrm{H} \beta$ upper limit give a lower limit on the extinction of $A_{V}=6.6$, which is consistent with both of the other methods to within the calibration uncertainty.

It is possible that the two measurements come from unresolved regions with different levels of extinction, though a factor of at least 3 change over an area $\sim 100 A U$ far from the millimeter cores seems unlikely. A strong IR radiation field could plausibly change the line ratio from the expected Einstein A value. The question is not resolved but may be possible to address with near-IR observations of nearby bright $\mathrm{HH}$ flows with more careful atmospheric calibration.

\subsection{Optical Spectra}

\subsubsection{Stellar Type}

IR 6 is suspected to be the source of the bright $\mathrm{H}_{2}$ finger at $\mathrm{PA} \approx 15^{\circ}$. IR 6 is also a $24 \mu m$ source and was detected by MSX (designation G173.4956+02.4218). We identify this star as a Herbig Ae/Be star.

\subsubsection{Density and Extinction Measurements}

The spectrum of knot N1 (the bow of Outflow 1) allowed a measurement of electron density in the shocks from the [S II] 6716/6731 line ratio . Densities were determined to be $n=700 \mathrm{~cm}^{-3}$ in the forward lump and $n=500 \mathrm{~cm}^{-3}$ in the second lump. $\mathrm{H} \alpha$, [N II] 6583, [O I] 6300, and [O III] 6363 were also detected, but no lines were detected in the blue portion of the spectrum presumably because of extinction. The measured velocities from [S II] are faster than the $\mathrm{H}_{2}$ velocity measurements at about $v_{L S R}=-68 \pm 5 \mathrm{~km} \mathrm{~s}^{-1}$.

There is also an ambient ionized medium that uniformly fills the slit with a [S II]-measured density $n_{e}=120 \mathrm{~cm}^{-3}$. Evidently, nearby massive stars are ionizing the low-density ISM located in front of IRAS 05358. This material is moving at velocity $v_{L S R}=-7 \pm 5 \mathrm{~km} \mathrm{~s}^{-1}$ and is extincted by $A_{V}=1.5$ as determined from $\mathrm{H} \alpha / \mathrm{H}_{\beta}=2.87$ assuming the gas is at $10^{4} \mathrm{~K}$. 


\subsection{UCHII region measurement}

A uniform density, ideal HII region will have an intensity curve $I=I_{0}\left(1-e^{-\tau_{\nu}}\right)$ where

$$
\tau=8.235 \times 10^{-2}\left(\frac{T_{e}}{K}\right)^{-1.35}\left(\frac{\nu}{\mathrm{GHz}}\right)^{-2.1}\left(\frac{\mathrm{EM}}{\mathrm{pc} \mathrm{cm}^{-6}}\right) a(\nu, T)
$$

following Rohlfs \& Wilson (2004) equation 9.35, where $a(\nu, T) \approx 1$ is a correction factor. By assuming an excitation temperature $T_{e x}=8500 \mathrm{~K}$, blackbody with a turnover to an optically thin thermal source was fit to the centimeter SED. The turnover frequency from this fit is $\tau=15.5 \mathrm{GHz}$, corresponding to an emission measure $E M=7.4 \times 10^{8} \mathrm{pc} \mathrm{cm}^{-6}$. This turnover frequency is lower than the $\sim 35 \mathrm{GHz}$ reported by Beuther et al. (2007). The turnover is clearly visible in the U, K and $\mathrm{Q}$ data points in figure 14 .

By assuming the X-band emission is optically thick, a source size can be derived.

$$
2 r=\left[\frac{S_{\nu}}{2 k_{B} T_{e x}} \lambda^{2} D^{2}\right]^{1 / 2}
$$

where D is the distance to the source. Assuming a spherical UCHII region and a distance of 1.8 $\mathrm{kpc}$, the source has radius $r=30 \mathrm{AU}$ (for comparison, the $\mathrm{Q}$ band beam minor axis is $\sim 90 \mathrm{AU}$, so the region could in principle be resolved by the VLA + Pie Town configuration).

The measured density is $n=(E M / r)^{1 / 2}=2.2 \times 10^{6} \mathrm{~cm}^{-3}$, with a corresponding emitting mass $M=n \mu m_{H} 4 / 3 \pi r^{3}=1.0 \times 10^{-6} M_{\odot}$ using $\mu=1.4$. Using Kurtz et al. (1994) equation 1,

$$
N_{L y c}=\left(8.04 \times 10^{46} s^{-1}\right) T_{e}^{-0.85}\left(\frac{r}{p c}\right)^{3} n_{e}^{2}
$$

the number of Lyman continuum photons per second required to maintain ionization is estimated to be $N_{L y c}=5.9 \times 10^{44}$, a factor of $\sim 4$ lower than measured by Beuther et al. (2007) and closer to a B2 ZAMS star $\left(\sim 11 M_{\odot}\right)$ than B1 using Table 2 of Panagia (1973). If the star has not yet reached the main sequence, it could be significantly more massive (Hosokawa \& Omukai 2009), so our stellar mass estimate is a lower limit.

The gravitational binding radius of a $11 M_{\odot}$ star is $r_{g}=2 G M / c_{s}^{2} \approx 190 \mathrm{AU}$ (the HII region is assumed to be supported entirely by thermal pressure, which provides an upper limit on the binding radius since turbulent pressure can exceed thermal pressure). The UCHII region radius of $30 \mathrm{AU}$ is much smaller, indicating that, under the assumption of spherical symmetry, the HII region is bound.

Leurini et al. (2007) noted that the $\mathrm{CH}_{3} \mathrm{CN}$ line profile around this source could be fit with a binary system with separation $<1100 \mathrm{AU}$ and a total mass of 7-22 $M_{\odot}$. This is entirely consistent with our picture of a massive binary system with a $11 M_{\odot}$ star in a UCHII region and another high mass star with a maser disk. 
There are no other sources in the IRAS 05358 region to a $5 \sigma$ limit of $0.075 \mathrm{mJy}$ in the X-band, which provides the strictest upper limit. From equation 3 , this corresponds to an optically thick source size of $24 \mathrm{AU}$. The maser disk has a spatial extent of around $140 \mathrm{AU}$, so it is quite unlikely that either an undetected UCHII region or the observed UCHII are associated with the maser disk.

Assuming the same turnover point for undetected sources, an upper limit is set on $N_{L y c}$ for undetected sources:

$$
N_{L y c}=\left(8.04 \times 10^{46} s^{-1}\right)\left(\frac{S_{\nu}}{2 k_{B} T_{e x}^{1.85}} \lambda_{c m}^{2} D_{p c}^{2}\right) E M
$$

Our $5 \sigma$ upper limit is $N_{L y c}=1.38 \times 10^{44} \mathrm{~s}^{-1}$, indicating that any stars present must be a later class than B3, or lower than about $8 M_{\odot}$. For an emission measure as much as 3 times higher, the corresponding stellar mass would be less than $10 M_{\odot}$. It is likely that no other massive stars have formed in Sh 2-233IR NE.

After independently determining the best-fit UCHII model to the VLA data, we included the PdBI data points from Beuther et al. (2007) and fit a power-law to both data sets. If the emission measure was allowed to vary, the derived parameters were $E M=6.3 \times 10^{8}$ and $\beta=0.7$. However, doing this visibly worsened the UCHII region fit without significantly improving the power-law fit, so the fit was repeated holding a fixed emission measure, yielding $\beta=0.8$ (plotted in Figure 14b). This power-law is much shallower than the $\beta=1.6$ measured by Beuther et al. (2007) without access to the $44 \mathrm{GHz}$ data point, and suggests that there is a significant population of large grains in source mm1a. However, we caution that the fits were performed only accounting for statistical errors, not the significant and unknown systematic errors that are likely to be present in mm interferometric data. The PdBI beams are much larger than the VLA beams, so the larger beams could be systematically shifted up by including additional emission, which would reduce $\beta$. Nonetheless, the new VLA data constrains the UCHII emission to contribute no more than $10 \%$ of the $3.1 \mathrm{~mm}$ flux.

\subsection{Mass, Energy, and Momentum estimates from CO}

\subsubsection{Equations}

The column density for $\mathrm{CO} \mathrm{J}=3-2$ is estimated using the equation

$$
N_{\mathrm{H}_{2}}=\frac{\mathrm{H}_{2}}{\mathrm{CO}} \frac{8 \pi \nu^{3} k_{B}}{3 c^{3} h B_{e} A_{u l}}\left(1-e^{h \nu / k_{B} T_{e x}}\right)^{-1} \frac{1}{\eta_{m b}} \int T_{A}^{*}(v) d v
$$

where $A_{u l}=A_{32}=2.5 \times 10^{-6} \mathrm{~s}^{-1}$ and $A_{21}=6.9 \times 10^{-7} \mathrm{~s}^{-1}$ (Turner et al. 1977), the rotational constant $B_{e}=57.64,55.10$, and $55.89 \mathrm{GHz}$ for ${ }^{12} \mathrm{CO},{ }^{13} \mathrm{CO}$, and $\mathrm{C}^{18} \mathrm{O}$ respectively, $\eta_{m b}=.68$, and $T_{e x}$ is assumed to be $20 \mathrm{~K}$. The partition function is approximated as

$$
Z=\sum_{J=1}^{\infty}(2 J+1) \exp \left(\frac{-J(J+1) h B_{e}}{k_{B} T_{e x}}\right) \approx \int_{0}^{\infty}(2 J+1) \exp \left(\frac{-J(J+1) h B}{k_{b} T_{e x}}\right) d J
$$



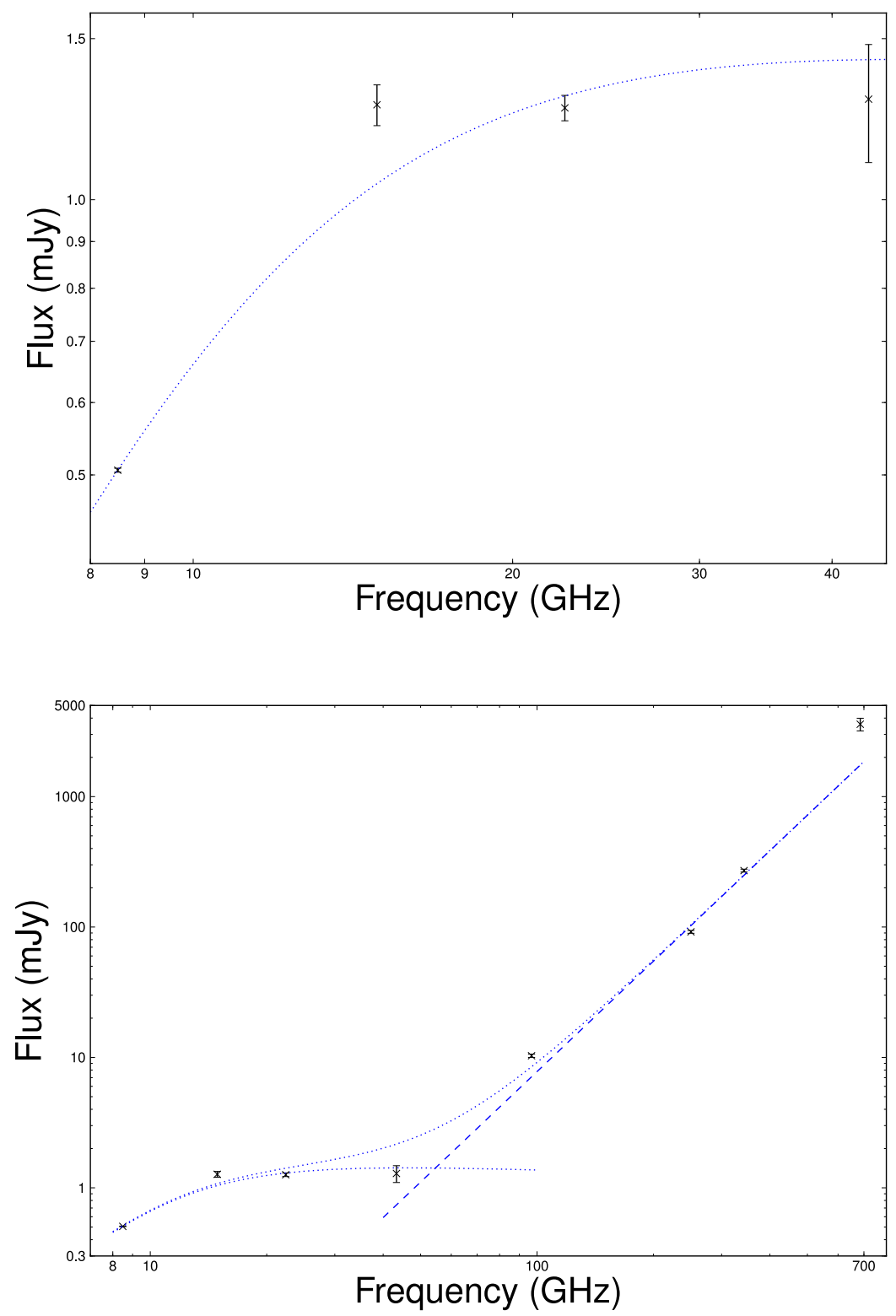

Fig. 14.- (a) The HII region fit to measured X, K, U, and Q band data. Error bars represent statistical error in the flux measurement. The $\mathrm{Q}$ band error is dominated by flux calibration uncertainty (see Table 1). The measured turnover is at $9.5 \mathrm{GHz}$. (b) A fit to both the VLA data presented in this paper and the (sub)mm points from Beuther et al. (2007). The best fit spectral index for the dust emission is $\alpha=2.8(\beta=0.8)$, significantly lower than the $\alpha=3.6$ measured by Beuther et al. (2007) without the $0.7 \mathrm{~mm}$ data point. 
which is valid when $T_{e x}>>h B_{e} / k_{B} \sim 2.8 \mathrm{~K}$. Equation 6 becomes

$$
N_{\mathrm{H}_{2}}=\left(3.27 \times 10^{18} \mathrm{~cm}^{-2}\right) \frac{1}{\eta_{m b}} \int T_{A}^{*}(v) d v
$$

where the integrand is in units $\mathrm{K} \mathrm{km} \mathrm{s}^{-1}$. The mass is then

$$
M=\mu m_{\mathrm{H}_{2}} A N_{\mathrm{H}_{2}}=1.42 \times 10^{-5} A \frac{1}{\eta_{m b}} \int T_{A}^{*}(v) d v
$$

where $\mathrm{A}$ is the area in $\mathrm{cm}^{2}, \mu=1.4$ is a constant to account for the presence of helium, and again velocity is in $\mathrm{km} \mathrm{s}^{-1}$.

\subsection{2. $C O J=2-1$ Isotopologue Comparison}

Thomas \& Fuller (2008) observed $\mathrm{C}^{17} \mathrm{O}$ in the $\mathrm{J}=2-1$ and $3-2$ transitions each with a single pointing using the JCMT centered at $\mathrm{J}(2000)=05: 39: 10.8+35: 45: 16$ and measured a column density $N_{\mathrm{H}_{2}}=4.03 \times 10^{22} \mathrm{~cm}^{-2}$. The peak column density is $1.7 \times 10^{22} \mathrm{~cm}^{-2}$ in ${ }^{13} \mathrm{CO}$ and $2.2 \times 10^{22}$ in $\mathrm{C}^{18} \mathrm{O}$ at $\mathrm{J}(2000)=5: 39: 10.2+35: 45: 26$, which is reasonably consistent with the $\mathrm{C}^{17} \mathrm{O}$ measurement considering abundance uncertainties. The peaks of the integrated spectra for $\mathrm{C}^{18} \mathrm{O}$ and ${ }^{13} \mathrm{CO}$ are coincident, but the ${ }^{12} \mathrm{CO}$ integrated peak is at $\mathrm{J}(2000)=5: 39: 12.6+35: 45: 46$ (Figure (7) discussed more in section [5.1).

Measurements of the column density, mass, momentum, and energy are performed as in Equation 6. Assuming a ${ }^{12} \mathrm{CO} /{ }^{13} \mathrm{CO}$ ratio of 60 (Lucas \& Liszt 1998) and optically thin ${ }^{13} \mathrm{CO}$, the mean column density across the region is $N_{\mathrm{H}_{2}}=4.5 \times 10^{21} \mathrm{~cm}^{-2}$. The resulting total mass of the central $\sim 3^{\prime}$ is about $320 M_{\odot}$, which is substantially smaller than the $600 M_{\odot}$ measured by Beuther et al. (2002a) and Zinchenko et al. (1997), but it is nearly consistent with $870 \mu \mathrm{m}$ and $\mathrm{NH}_{3}$ estimates of 450 and $400 M_{\odot}$ from Mao \& Zeng (2004) and is within the systematic uncertainties of these measurements. Assuming $\mathrm{C}^{18} \mathrm{O}$ is optically thin and the $\mathrm{C}^{18} \mathrm{O} /{ }^{13} \mathrm{CO}$ ratio is 10 , the column density is $5.2 \times 10^{21} \mathrm{~cm}^{-2}$ and the mass is $360 M_{\odot}$, which is consistent with the ${ }^{13} \mathrm{CO}$ measurements, indicating that optical depth effects are probably not responsible for the discrepancy with the dust mass estimate.

\subsubsection{CO Mass and Energy Measurements for Specific Outflows}

Table 2 lists measurements of mass and momentum in apertures shown in figure 6. Where red and blue masses are listed, there is an outflow in the red and blue along the line of sight. Where only one is listed, an excess to one side of the cloud rest velocity was detected and assumed to be accelerated gas from a protostellar outflow. Blue velocities are integrated from -33 to $-21 \mathrm{~km} \mathrm{~s}^{-1}$. Red velocities are integrated from $-12 \mathrm{~km} \mathrm{~s}^{-1}$ to $1 \mathrm{~km} \mathrm{~s}^{-1}$. All masses are computed assuming CO is optically thin in the outflow, which leads to a lower bound on the mass; ${ }^{13} \mathrm{CO} 2-1$ was measured 
to have an optical depth of 0.1 in 7 very high velocity outflows in Choi et al. (1993), so if a relative abundance ${ }^{12} \mathrm{CO} /{ }^{13} \mathrm{CO}=60$ is assumed(Lucas \& Liszt 1998), masses increase by a factor of 6 .

It is not possible to completely distinguish outflowing matter from the ambient medium. While the outflowing matter is generally at higher velocities, the outflow and ambient line profiles are blended. A uniform selection of high velocities was applied across the region but this may include some matter from the cloud, biasing the mass measurements upward. Outflows in the plane of the sky and low-velocity components of outflows will be blended with the cloud profile, which would lead to underestimates of the outflowing mass. The momentum measurements, however, should be more robust because they are weighted by velocity, and higher velocity material is more certainly outflowing. The momentum measurements are referenced to the central velocity of Sh 2-233IR NE, $v_{L S R}=-16.0 \mathrm{~km} \mathrm{~s}^{-1}$.

\section{Discussion}

\subsection{Outflow Mass and Momentum}

Beuther et al. (2002a) reported a total outflowing mass of $20 M_{\odot}$ in Sh 2-233IR NE. We measure a significantly lower outflow mass of $2 M_{\odot}$ under the assumption that the gas is optically thin, but this assumption is not valid: a lower limit can be set from the weak ${ }^{13} \mathrm{CO} 2-1$ outflow detection (lower limit because not all of the outflowing material is detected) on the outflowing mass of $\sim 4 M_{\odot}$. Choi et al. (1993) measure an optical depth of ${ }^{13} \mathrm{CO} 2-1 \tau \approx 0.1$ in 7 very high velocity outflows. Our ${ }^{13} \mathrm{CO}$ data suggests that the optical depth is somewhat lower, $\tau \approx 0.07$. The abundance ${ }^{12} \mathrm{CO} /{ }^{13} \mathrm{CO}=60$ is used (Lucas \& Liszt 1998) to derive a total outflowing mass estimate $M \approx 25 M_{\odot}$. The total outflowing mass is therefore $\sim 4 \%$ of the total cloud mass, though most of the outflowing material is coming from Sh 2-233IR NE, so as much as $13 \%$ of the material in Sh 2-233IR NE may be outflowing.

The most prominent outflow in IRAS 05358, Outflow 1, is primarily along the plane of the sky, so the high velocity $\mathrm{CO}$ is likely associated with the other outflows that have significant components along the line of sight. As pointed out in Beuther et al. (2002a), the integrated and peak CO are aligned with the main $\mathrm{mm}$ core. High-velocity $\mathrm{H}_{2}$ near the $\mathrm{mm}$ cores and the blueshifted outflows 2 and 4 all suggest that there are many distinct outflows that together are responsible for the high velocity $\mathrm{CO}$ gas.

The offset between the integrated ${ }^{13} \mathrm{CO}$ peak and ${ }^{12} \mathrm{CO}$ peak in the $\mathrm{J}=2-1$ integrated maps, which corresponds with an offset in the peak of the integrated CO 3-2 map and the peak temperature observed in CO 3-2, suggests that the gas mass is largely associated with Sh 2-233IR SW, but the outflowing gas is primarily associated with Sh 2-233IR NE. The integrated and maximum brightness temperatures in ${ }^{13} \mathrm{CO}$ and $\mathrm{C}^{18} \mathrm{O}$ are also centered near Sh 2-233IR SW, which rules out optical depth as the cause of this offset. CO may be depleted in the dense mm cores, which would help account 
for the lower mass estimate from $\mathrm{CO}$ isotopologues relative to dust mass and $\mathrm{NH}_{3}$. Alternately, the gas temperature in Sh 2-233IR SW may be significantly higher than in Sh 2-233IR NE except in the outflows, which are probably warm. In this case, the outflowing ${ }^{12} \mathrm{CO}$ enhances the integrated intensity because of its high temperature and reduced effective optical depth, but it does not set the peak brightness because of the low filling-factor of the high-temperature gas.

Because the outflows are seen in $\mathrm{H}_{2}$, which requires shock velocities $\sim 30 \mathrm{~km} \mathrm{~s}^{-1}$ to be excited (Bally et al. 2007), and because the association between the high-velocity CO and the plane-of-thesky $\mathrm{H}_{2}$ is unclear, a velocity of $30 \mathrm{~km} \mathrm{~s}^{-1}$ is used when estimating the dynamical age. Assuming the outflow is about $0.5 \mathrm{pc}$ long in one direction (e.g. Outflow 1), the dynamical age is $1.6 \times 10^{4}$ years. Outflow 4, which is around $1 \mathrm{pc}$ long, is also seen at a velocity of $-70 \mathrm{~km} \mathrm{~s}^{-1} \mathrm{LSR}$, or about -50 $\mathrm{km} \mathrm{s}^{-1}$ with respect to the cloud, and therefore has a dynamic age $2 \times 10^{4}$ years, which is consistent.

\subsection{Energy Injection / Ejection}

Using an assumed outflow lifetime of $5 \times 10^{3}$ years for $v=100 \mathrm{~km} \mathrm{~s}^{-1}$ as a lower limit (because the full extent of the flows is not necessarily observed) and $1 \times 10^{5}$ as an upper limit (for the CO velocities $\sim 10 \mathrm{~km} \mathrm{~s}^{-1}$ and the longest $\sim 1 \mathrm{pc}$ flows), mechanical luminosities of the outflows $L=E / t$ are derived. The summed mechanical luminosity of the outflows is compared to the turbulent decay luminosity within a $12^{\prime \prime}, 1^{\prime}$, and $5^{\prime}$ radius centered on Sh 2-233IR NE in Table 9.

The rate of turbulent decay can be estimated from the crossing-time of the region, $L / v$, where $L$ is the length scale and $v$ is the the typical turbulent velocity. On the largest ( $\sim$ few pc) scales, the mechanical luminosity from high-velocity outflowing material is approximately capable of balancing turbulent decay and upholding the cloud against collapse. However, at the size scales of the Sh 2233IR NE clump ( $\sim 0.1 \mathrm{pc})$, turbulent decay occurs on more than an order of magnitude faster timescales than outflow energy injection. On the smallest scales, outflow energy can be lost from the cluster through collimated outflows, though wide-angle flows and wrapped up magnetic fields

Table 9. Comparison of turbulent decay and outflow injection

\begin{tabular}{ccccccc}
\hline \hline Radius $(\mathrm{pc})$ & $t_{\text {turb }}{ }^{\mathrm{a}}(\mathrm{yr})$ & $L_{\text {turb }}\left(L_{\odot}\right)$ & $L_{\text {outflows }}{ }^{\mathrm{b}}\left(L_{\odot}\right)$ & Binding Energy $(\mathrm{ergs}){ }^{\mathrm{c}}$ Outflow Energy $(\mathrm{ergs})$ & Turbulent Energy $(\mathrm{ergs})$ \\
\hline 0.10 & $2 \times 10^{4}$ & 20 & $0.03-0.6$ & $3.4 \times 10^{46}$ & $3.5 \times 10^{44}$ & $5.0 \times 10^{46}$ \\
0.52 & $1 \times 10^{5}$ & 12 & $0.6-9.4$ & $5.9 \times 10^{46}$ & $5.9 \times 10^{45}$ & $1.5 \times 10^{47}$ \\
2.62 & $5 \times 10^{5}$ & 2.3 & $1-22$ & $1.2 \times 10^{46}$ & $1.4 \times 10^{46}$ & $1.5 \times 10^{47}$ \\
\hline
\end{tabular}

${ }^{\text {a }}$ Masses are assumed to be $600 M_{\odot}$ for the $1^{\prime}$ and $5^{\prime}$ apertures, and $200 M_{\odot}$ for the $12^{\prime \prime}$ aperture.

${ }^{\mathrm{b}}$ Outflow luminosities are given as a range with a lower limit $L=E_{\text {out }} / 10^{5} \mathrm{yr}$ and upper limit $L=E_{\text {out }} / 5 \times 10^{3} \mathrm{yr}$, where $E_{\text {out }}$ is from Table 2 multiplied by 6 to correct for outflow opacity.

${ }^{\mathrm{c}}$ Binding energy is the order-of-magnitude estimate $\mathrm{GM}^{2} / \mathrm{R}$

$\mathrm{d}$ Turbulent energy is computed using the measured $5 \mathrm{~km} \mathrm{~s}^{-1}$ line width as the turbulent velocity. 
will not propagate outside of the core region. Once collimated flows impact the local interstellar medium in a bow shock, their energy and momentum are distributed more isotropically and again contribute to turbulence. The imbalance on a small size scale is consistent with the observed infall signature (Figure 9) in the inner 12" around Sh 2-233IR NE and the lack of a similar profile elsewhere.

\subsection{Comparison to other clumps}

The classification scheme laid out in Klein et al. (2005) is used to identify Sh 2-233IR NE as a Protocluster and Sh 2-233IR SW as a Young Cluster. Maury et al. (2009) performed a similar analysis of the Early Protocluster NGC 2264-C. They also found that the outflow mechanical luminosity could provide the majority of the turbulent energy $L_{t u r b} \sim 1.2 L_{\odot}$ within the protocluster in a radius of $0.7 \mathrm{pc}$ with a mass $2300 M_{\odot}$. Williams et al. (2003) performed an outflow study of the OMC 2/3 region with radius $1.2 \mathrm{pc}$ and mass $1100 M_{\odot}$, which is also an Early Protocluster, and concluded that $L_{\text {turb }} \sim L_{\text {flow }} \sim 1.3 L_{\odot}$. While all three regions have nearly the same turbulent decay luminosities and outflow mechanical luminosities, Sh 2-233IR NE in IRAS 05358 is significantly more compact and lower mass than the Early Clusters, and is the only one of the three that contains signatures of massive star formation.

\subsection{Surrounding Regions}

About $8^{\prime}$ to the southeast of IRAS 05358 is another embedded star forming region, G173.58+2.45. Interferometric and stellar population studies have been performed by Shepherd \& Churchwell (1996) and Shepherd \& Watson (2002). The bipolar outflow detected in their interferometric maps is also cleanly resolved in our figure 6. In our wide-field $\mathrm{H}_{2}$ maps, there is a complex of outflows similar to that of IRAS 05358, but fainter.

The large HII region Sharpless 231 to the northeast can be seen in the H $\alpha$ image (figure 1). The expanding HII region is pushing against the molecular ridge that includes IRAS 05358 and accelerating the $\mathrm{CO}$ gas in the blue direction (e.g. the northern blueshifted clumps in figures 6 and (5). It can be seen from the IRAC $8 \mu m$ data that UV radiation from the HII region reaches to the IRAS 05358 clusters. The expanding HII region's pressure on the molecular ridge may be responsible for triggering the collapse of IRAS 05358 and G173. The size gradient from S232 ( 30' across) to S231 $\left(\sim 10^{\prime}\right)$ to S233 $\left(\sim 2-3^{\prime}\right)$ is suggestive of an age gradient assuming uniform HII region expansion velocities and a common distance. Investigation of this hypothesis will require detailed stellar population studies in the HII regions with proper regard for eliminating foreground and background sources. 


\subsection{Massive Star Binary}

Our identification of a probable massive star binary with an associated outflow contributes to a very small sample of known maser disks with $\mathrm{H}_{2}$ emission perpendicular to the disk. De Buizer (2003) observed 28 methanol maser sources with linear distributions of maser spots in the $\mathrm{H}_{2} 2.12$ $\mu m$ line, and he identified only 2 sources with $\mathrm{H}_{2}$ emission perpendicular to the maser lines. None of the outflows identified in his survey were as collimated as Outflow 2, so the methanol disk / outflow combination presented here may be the most convincing association of a massive protostellar disk with a collimated outflow.

The association of a massive star with an UCHII region and a methanol maser disk and the very small size of the UCHII region both suggest that the massive stellar system is very young. Walsh et al. (1998) suggested that the development of a UCHII region leads to the destruction of maser emission regions. Their conclusion is consistent with our interpretation of mm1a as a binary system.

\section{Summary \& Conclusion}

We have presented a multiwavelength study of the IRAS 05358 star forming region. IRAS 05358 contains an embedded cluster of massive stars and is surrounded by outflows. The outflows were linked to probable sources and determined that at least one outflow is probably associated with a massive $\left(\sim 10 M_{\odot}\right)$ star. Added kinematic information and a wide field view of the infrared outflows has been used to develop a more complete picture of the region.

- Sh 2-233IR NE is a Protocluster and Sh 2-233IR SW is a Young Cluster

- Energy injection on the scales of IRAS 05358 can maintain turbulence, but on the small scales of the Sh 2-233IR NE protocluster, is inadequate by $\sim 2$ orders of magnitude. Sh 2-233IR NE is collapsing.

- there are 11 candidate outflows, 7 of which have candidate counterflows, in the IRAS 05358 complex

- there is a probable massive binary with one member of mass $12 M_{\odot}$ in mm1a, and the other which is the source of Outflow 2

- there are at least two moderate-mass $\left(\sim 5 M_{\odot}\right)$ young stars in IRAS 05358

We have identified additional middle- and high-mass young stars with outflows, and presented a case for a high-mass binary system within the millimeter core mm1a. 


\section{Acknowledgements}

We would like to thank Vincent Minier for providing us with the positions of the VLBI maser spots and Steve Myers and George Moellenbrock for their assistance with VLA data reduction.

We would also like to thank Cara Battersby, Devin Silvia, Mike Shull, and Jeremy Darling for helpful comments on early drafts.

This work made use of SAOIMAGE DS9 (http://hea-www.harvard.edu/RD/ds9/), IRAF (http://iraf.net/, scipy (http://www.scipy.org), and APLpy (http://aplpy.sourceforge.net/).

\section{REFERENCES}

Alvarez, C. \& Hoare, M. G. 2005, A\&A, 440, 569

Bally, J., Reipurth, B., \& Davis, C. J. 2007, in Protostars and Planets V, ed. B. Reipurth, D. Jewitt, \& K. Keil, 215-230

Beuther, H., Leurini, S., Schilke, P., Wyrowski, F., Menten, K. M., \& Zhang, Q. 2007, A\&A, 466, 1065

Beuther, H., Schilke, P., Gueth, F., McCaughrean, M., Andersen, M., Sridharan, T. K., \& Menten, K. M. 2002a, A\&A, 387, 931

Beuther, H., Schilke, P., Sridharan, T. K., Menten, K. M., Walmsley, C. M., \& Wyrowski, F. 2002b, A\&A, 383, 892

Casoli, F., Combes, F., Dupraz, C., Gerin, M., \& Boulanger, F. 1986, A\&A, 169, 281

Choi, M., Evans, II, N. J., \& Jaffe, D. T. 1993, ApJ, 417, 624

De Buizer, J. M. 2003, MNRAS, 341, 277

Giannini, T., Calzoletti, L., Nisini, B., Davis, C. J., Eislöffel, J., \& Smith, M. D. 2008, A\&A, 481, 123

Henning, T., Cesaroni, R., Walmsley, M., \& Pfau, W. 1992, A\&AS, 93, 525

Heyer, M. H., Carpenter, J. M., \& Ladd, E. F. 1996, ApJ, 463, 630

Hosokawa, T. \& Omukai, K. 2009, ApJ, 691, 823

Jiang, Z., Yao, Y., Yang, J., Ishii, M., Nagata, T., Nakaya, H., \& Sato, S. 2001, AJ, 122, 313

Khanzadyan, T., Smith, M. D., Davis, C. J., \& Stanke, T. 2004, A\&A, 418, 163

Klein, R., Posselt, B., Schreyer, K., Forbrich, J., \& Henning, T. 2005, ApJS, 161, 361 
Krumholz, M. R., Klein, R. I., McKee, C. F., Offner, S. S. R., \& Cunningham, A. J. 2009, Science, 323,754

Kumar, M. S. N., Bachiller, R., \& Davis, C. J. 2002, ApJ, 576, 313

Kurtz, S., Churchwell, E., \& Wood, D. O. S. 1994, ApJS, 91, 659

Lada, C. J. \& Lada, E. A. 2003, ARA\&A, 41, 57

Leurini, S., Beuther, H., Schilke, P., Wyrowski, F., Zhang, Q., \& Menten, K. M. 2007, A\&A, 475, 925

Longmore, S. N., Burton, M. G., Minier, V., \& Walsh, A. J. 2006, MNRAS, 369, 1196

Lucas, R. \& Liszt, H. 1998, A\&A, 337, 246

Luhman, K. L., Engelbracht, C. W., \& Luhman, M. L. 1998, ApJ, 499, 799

Mao, R.-Q. \& Zeng, Q. 2004, Chinese Journal of Astronomy and Astrophysics, 4, 440

Maury, A. J., André, P., \& Li, Z.-Y. 2009, A\&A, 499, 175

Menten, K. M. 1991, ApJ, 380, L75

Minier, V., Booth, R. S., \& Conway, J. E. 2000, A\&A, 362, 1093

Osterbrock, D. E. \& Ferland, G. J. 2006, Astrophysics of gaseous nebulae and active galactic nuclei, ed. D. E. Osterbrock \& G. J. Ferland

Panagia, N. 1973, AJ, 78, 929

Parker, N. D., Padman, R., \& Scott, P. F. 1991, MNRAS, 252, 442

Porras, A., Cruz-González, I., \& Salas, L. 2000, A\&A, 361, 660

Qiu, K. et al. 2008, ApJ, 685, 1005

Reipurth, B. \& Bally, J. 2001, ARA\&A, 39, 403

Robitaille, T. P., Whitney, B. A., Indebetouw, R., \& Wood, K. 2007, ApJS, 169, 328

Rohlfs, K. \& Wilson, T. L. 2004, Tools of radio astronomy (Tools of radio astronomy, 4th rev. and enl. ed., by K. Rohlfs and T.L. Wilson. Berlin: Springer, 2004)

Scalise, Jr., E., Rodriguez, L. F., \& Mendoza-Torres, E. 1989, A\&A, 221, 105

Schöier, F. L., van der Tak, F. F. S., van Dishoeck, E. F., \& Black, J. H. 2005, A\&A, 432, 369

Shepherd, D. S. \& Churchwell, E. 1996, ApJ, 457, 267 
Shepherd, D. S. \& Watson, A. M. 2002, ApJ, 566, 966

Smith, N. \& Hartigan, P. 2006, ApJ, 638, 1045

Thomas, H. S. \& Fuller, G. A. 2008, A\&A, 479, 751

Turner, J., Kirby-Docken, K., \& Dalgarno, A. 1977, ApJS, 35, 281

Valdes, F. G. \& Swaters, R. A. 2007, in Astronomical Society of the Pacific Conference Series, Vol. 376, Astronomical Data Analysis Software and Systems XVI, ed. R. A. Shaw, F. Hill, \& D. J. Bell, 273-+

Walsh, A. J., Burton, M. G., Hyland, A. R., \& Robinson, G. 1998, MNRAS, 301, 640

Wang, H., Yang, J., Wang, M., \& Yan, J. 2003, AJ, 125, 842

Williams, J. P., Plambeck, R. L., \& Heyer, M. H. 2003, ApJ, 591, 1025

Williams, S. J., Fuller, G. A., \& Sridharan, T. K. 2004, A\&A, 417, 115

Wouterloot, J. G. A., Brand, J., \& Fiegle, K. 1993, A\&AS, 98, 589

Yan, C. 2009, ApJ

Zinchenko, I., Henning, T., \& Schreyer, K. 1997, A\&AS, 124, 385 